\title{
On the Existence of Leapfrogging Pair of Circular Vortex Filaments
}

\author{
Masashi AIKI
}

\begin{abstract}
We propose and analyze a system of nonlinear partial differential equations describing the motion of a pair of vortex filaments. Furthermore, for a pair of coaxial circular vortex filaments, we derive a condition for leapfrogging to occur and prove that the condition is necessary and sufficient for the occurrence of leapfrogging.
\end{abstract}

\section{Introduction and Problem Setting}

In this paper, we are interested in the interaction of two vortex rings sharing the same axis of symmetry. The study of the interaction of two vortex rings dates back to the classical paper by Helmholtz [8], where he observed that a pair of vortex rings may exhibit what is now known as "leapfrogging". Leapfrogging is a motion pattern where two vortex rings sharing a common axis of symmetry pass through each other repeatedly due to the induced flow of the rings acting on each other. Dyson $[5,6]$ considered the motion of vortex rings and proposed a system of equations describing the motion of a pair of coaxial vortex rings. Based on this model system, Dyson also observed that leapfrogging may occur. Hicks [9] also considered the interaction of a pair of vortex rings and derived a model similar to the one obtained by Dyson. He also made numerical observations that depending on the vorticity strengths and initial configuration of the rings, the pair may show leapfrogging, or the two rings may separate indefinitely. Although these observations were known for a long time, the first experiment which successfully provided photographic proofs of the leapfrogging phenomenon in a laboratory setting was conducted by Yamada and Matsui [17]. They used vortex rings made of air and used smoke to visualize the rings and created a leapfrogging pair of rings, and hence, leapfrogging vortex rings were observed in the real world. In recent years, detailed models for the motion of vortex rings have been obtained, for example by Saffman [15] and Fukumoto [7], in which various distributions of vorticity in the ring core and the change in shape of the core can be incorporated. Based on these models, Borisov, Kilin, and Mamaev [2] gave a complete description of the possible motion patterns of two interacting vortex rings with a common axis of symmetry. Shariff and Leonard [16] give a review of the history of the research of vortex rings in which many other phenomena related to the motion of single and multiple vortex rings, including the leapfrogging phenomenon, are addressed. 
The study of the motion of a vortex ring, and the interaction of vortex rings are not only interesting from a theoretical stand point, but also plays an important role in engineering. One such example being the generation of sound from a round jet as studied in Crighton [3], Hussain and Zaman [11], and Zaman [18].

The aim of the present paper is to propose and analyze a system of nonlinear partial differential equations for the motion of two interacting vortex filaments. A vortex filament is a space curve on which the vorticity of the fluid is concentrated. A vortex filament can be seen as an idealization of a thin vortex structure for which the evolution can be approximated by the evolution of its center line. A model equation, known as the Localized Induction Equation, describing the motion of a single vortex filament was first proposed by Da Rios [4] and later independently derived by Murakami et al. [13] and Arms and Hama [1]. The main idea used in these papers is the so-called localized induction approximation, and we follow this concept in this paper to derive a system of equations describing the motion of two interacting vortex filaments. We further consider the case when the filaments are circular with a common axis of symmetry and obtain a necessary and sufficient condition for leapfrogging to occur.

The motivation for this work is the following. Many of the analysis made for leapfrogging vortex rings are conducted for a system of ordinary differential equations. This, of course, is natural since for a coaxial vortex ring pair, the circular shape is expected to be preserved from the symmetry of the induced flow. On the other hand, when one considers stability of such motion, it would be useful if the motion is described in the framework of partial differential equations because it becomes possible to consider non-symmetric perturbations. Consequently, this complicates the situation and thus, we consider circular vortex filaments instead of vortex rings with finite core thickness. As far as the author knows, the model proposed by Klein, Majda, and Damodaran [12] is the only model describing the motion of multiple vortex filaments in the framework of partial differential equations. They consider filaments that are nearly straight and parallel to each other and derive a model system describing their motion, and as such, the motion described by this model is two-dimensional. Hence, when considering leapfrogging circular filaments, the model in [12] is not suitable, and we derive a different system in this paper.

The rest of the paper is organized as follows. In Section 2, we derive the model system via the localized induction approximation. We also give some exact solutions of the obtained system to show that the model is capable of describing well known motions of straight vortex filaments which are parallel to each other. In Section 3, we consider the case when the two filaments are circular with a common axis of symmetry and the vorticity strengths have the same sign. We show that the problem can be reduced to a two-dimensional Hamiltonian system and give a necessary and sufficient condition for leapfrogging to occur. The precise statement will be given in the beginning of Section 3. In Section 4, the leapfrogging phenomenon will be considered for a pair of circular filaments which have vorticity strengths of opposite signs. Similar to Section 3, we give a necessary and sufficient condition for leapfrogging to occur. Finally in Section 5, we compare our results with the results obtained in Borisov, Kilin, and Mamaev [2], and also give concluding remarks. 


\section{Interaction of Two Vortex Filaments}

We consider the interaction of two vortex filaments and derive a system of nonlinear partial differential equations which describe their motion. The obtained model admits solutions which correspond to well known motions of point vortices when the two filaments are straight parallel lines, and also gives a clear view of the dynamics when the filaments are arranged as coaxial circles, and hence the author hopes that the model could be of some significance.

\subsection{Derivation of the Model System}

Following the work of Arms and Hama [1], we apply the localized induction approximation to the Biot-Savart law to obtain a system of partial differential equations approximating the motion of two interacting vortex filaments. The velocity $\boldsymbol{v}(\boldsymbol{x})$ at some point $\boldsymbol{x} \in \mathbf{R}^{3}$ of an infinite body of incompressible and inviscid fluid induced by a pair of vortex filaments whose positions are parametrized by $\xi \in J$ at time $t \geq 0$ as $\boldsymbol{X}(\xi, t)$ and $\boldsymbol{Y}(\xi, t)$ is given by

$$
\boldsymbol{v}(\boldsymbol{x})=\frac{\Gamma_{1}}{4 \pi} \int_{J} \frac{\boldsymbol{X}_{\xi}(r, t) \times(\boldsymbol{x}-\boldsymbol{X}(r, t))}{|\boldsymbol{x}-\boldsymbol{X}(r, t)|^{3}} \mathrm{~d} r+\frac{\Gamma_{2}}{4 \pi} \int_{J} \frac{\boldsymbol{Y}_{\xi}(r, t) \times(\boldsymbol{x}-\boldsymbol{Y}(r, t))}{|\boldsymbol{x}-\boldsymbol{Y}(r, t)|^{3}} \mathrm{~d} r
$$

where $x$ is the exterior product in the three-dimensional Euclidean space, $\Gamma_{1}$ is the vorticity strength of the filament $\boldsymbol{X}, \Gamma_{2}$ is the vorticity strength of the filament $\boldsymbol{Y}$, $J=\mathbf{R}$ or $\mathbf{R} / 2 \pi \mathbf{Z}$, and subscripts denote the partial differentiation with the respective variables. The above equation is the Biot-Savart law when the vorticity is concentrated on two vortex filaments. The case $J=\mathbf{R}$ corresponds to when $\boldsymbol{X}$ and $\boldsymbol{Y}$ are infinitely long filaments, and the case $J=\mathbf{R} / 2 \pi \mathbf{Z}$ corresponds to when $\boldsymbol{X}$ and $\boldsymbol{Y}$ are closed filaments. To determine the velocity of a point on one of the filaments (say $\boldsymbol{X}(\xi, t)$ ), one would like to substitute $\boldsymbol{x}=\boldsymbol{X}(\xi, t)$ in equation (2.1), but this would result in the divergence of the first integral on the right-hand side. Hence we apply the localized induction approximation to approximate the the velocity at $\boldsymbol{X}(\xi, t)$ by the following equation.

$$
\begin{aligned}
\boldsymbol{v}(\boldsymbol{X}(\xi, t))= & \frac{\Gamma_{1}}{4 \pi} \int_{\varepsilon \leq|\xi-r| \leq L} \frac{\boldsymbol{X}_{\xi}(r, t) \times(\boldsymbol{X}(\xi, t)-\boldsymbol{X}(r, t))}{|\boldsymbol{X}(\xi, t)-\boldsymbol{X}(r, t)|^{3}} \mathrm{~d} r \\
& \quad+\frac{\Gamma_{2}}{4 \pi} \int_{|\xi-r| \leq \delta} \frac{\boldsymbol{Y}_{\xi}(r, t) \times(\boldsymbol{X}(\xi, t)-\boldsymbol{Y}(r, t))}{|\boldsymbol{X}(\xi, t)-\boldsymbol{Y}(r, t)|^{3}} \mathrm{~d} r \\
=: & I_{1}+I_{2} .
\end{aligned}
$$

Here, $\varepsilon>0$ and $\delta>0$ are small parameters, and $L>0$ is a cut-off parameter. $I_{1}$ is the effect of self-induction, and $I_{2}$ is the effect of interaction. The approximation applied in $I_{1}$ is the well known localized induction approximation. To obtain $I_{2}$, we have further assumed that the filaments $\boldsymbol{X}$ and $\boldsymbol{Y}$ are positioned in a way that $\boldsymbol{Y}(\xi, t)$ is the closest point to $\boldsymbol{X}(\xi, t)$ and the contributions from points far away from $\boldsymbol{Y}(\xi, t)$ can be ignored. 
This kind of geometric assumption is true for the situations that we treat in this paper, but does not hold, for example, when the filaments are knotted together. By the calculations in Arms and Hama [1], it is known that $I_{1}$ can be expanded in terms of small $\varepsilon$ as follows.

$$
I_{1}=-\frac{\Gamma_{1}}{4 \pi} \log \left(\frac{L}{\varepsilon}\right) \frac{\boldsymbol{X}_{\xi} \times \boldsymbol{X}_{\xi \xi}}{\left|\boldsymbol{X}_{\xi}\right|^{3}}+O(1) .
$$

The above is obtained by substituting the Taylor expansion of $\boldsymbol{X}(r, t)$ and $\boldsymbol{X}_{\xi}(r, t)$ with respect to $r$ around $\xi$ into the integrand. We further substitute

$$
\begin{gathered}
\boldsymbol{Y}(r, t)=\boldsymbol{Y}(\xi, t)+\boldsymbol{Y}_{\xi}(\xi, t)(r-\xi)+O\left((r-\xi)^{2}\right), \\
\boldsymbol{Y}_{\xi}(r, t)=\boldsymbol{Y}_{\xi}(\xi, t)+\boldsymbol{Y}_{\xi \xi}(\xi, t)(r-\xi)+O\left((r-\xi)^{2}\right),
\end{gathered}
$$

into $I_{2}$ to obtain

$$
I_{2}=\frac{\delta \Gamma_{2}}{2 \pi} \frac{\boldsymbol{Y}_{\xi} \times(\boldsymbol{X}-\boldsymbol{Y})}{|\boldsymbol{X}-\boldsymbol{Y}|^{3}}+O\left(\delta^{2}\right) .
$$

Hence, after fixing $L$ and taking sufficiently small $\varepsilon$ and $\delta$, the leading order terms of $I_{1}$ and $I_{2}$ yield

$$
\boldsymbol{X}_{t}=-\frac{\Gamma_{1}}{4 \pi} \log \left(\frac{L}{\varepsilon}\right) \frac{\boldsymbol{X}_{\xi} \times \boldsymbol{X}_{\xi \xi}}{\left|\boldsymbol{X}_{\xi}\right|^{3}}+\frac{\delta \Gamma_{2}}{2 \pi} \frac{\boldsymbol{Y}_{\xi} \times(\boldsymbol{X}-\boldsymbol{Y})}{|\boldsymbol{X}-\boldsymbol{Y}|^{3}},
$$

where we also used the fact that $\boldsymbol{v}(\boldsymbol{X}(\xi, t))=\boldsymbol{X}_{t}(\xi, t)$ by the definition of velocity. By rescaling time by a factor of $-\log \left(\frac{L}{\varepsilon}\right) / 4 \pi$, we obtain

$$
\boldsymbol{X}_{t}=\Gamma_{1} \frac{\boldsymbol{X}_{\xi} \times \boldsymbol{X}_{\xi \xi}}{\left|\boldsymbol{X}_{\xi}\right|^{3}}-\alpha \Gamma_{2} \frac{\boldsymbol{Y}_{\xi} \times(\boldsymbol{X}-\boldsymbol{Y})}{|\boldsymbol{X}-\boldsymbol{Y}|^{3}},
$$

where $\alpha=2 \delta / \log \left(\frac{L}{\varepsilon}\right)>0$. The calculations for the velocity at points on $\boldsymbol{Y}$ are the same and hence we arrive at the following system.

$$
\left\{\begin{array}{c}
\boldsymbol{X}_{t}=\Gamma_{1} \frac{\boldsymbol{X}_{\xi} \times \boldsymbol{X}_{\xi \xi}}{\left|\boldsymbol{X}_{\xi}\right|^{3}}-\alpha \Gamma_{2} \frac{\boldsymbol{Y}_{\xi} \times(\boldsymbol{X}-\boldsymbol{Y})}{|\boldsymbol{X}-\boldsymbol{Y}|^{3}} \\
\boldsymbol{Y}_{t}=\Gamma_{2} \frac{\boldsymbol{Y}_{\xi} \times \boldsymbol{Y}_{\xi \xi}}{\left|\boldsymbol{Y}_{\xi}\right|^{3}}-\alpha \Gamma_{1} \frac{\boldsymbol{X}_{\xi} \times(\boldsymbol{Y}-\boldsymbol{X})}{|\boldsymbol{X}-\boldsymbol{Y}|^{3}}
\end{array}\right.
$$

All the analysis that follows will be based on the above system (2.2).

\subsection{Dynamics of Two Parallel Lines}

As a preliminary analysis, we show that for a pair of infinitely long, straight, and parallel vortex filaments, the dynamics of the filaments according to equation (2.2) are the same 
as that of two point vortices moving in a plane. Suppose the two filaments are initially parametrized as

$$
\boldsymbol{X}_{0}(\xi)={ }^{t}(l, 0, \xi), \quad \boldsymbol{Y}_{0}(\xi)={ }^{t}(-l, 0, \xi)
$$

where $l>0$ is arbitrary. In this situation, it is expected that the motions of the filaments become two-dimensional and resemble that of two point vortices. Indeed, if we make the ansatz

$$
\boldsymbol{X}(\xi, t)={ }^{t}\left(x_{1}(t), x_{2}(t), \xi\right), \quad \boldsymbol{Y}(\xi, t)={ }^{t}\left(y_{1}(t), y_{2}(t), \xi\right)
$$

and substitute it into (2.2), we obtain

$$
\left\{\begin{array}{l}
\dot{x_{1}}=\frac{\alpha \Gamma_{2}\left(x_{2}-y_{2}\right)}{\left(\left(x_{1}-x_{1}\right)^{2}+\left(x_{2}-y_{2}\right)^{2}\right)^{3 / 2}}, \\
\dot{x_{2}}=-\frac{\alpha \Gamma_{2}\left(x_{1}-y_{1}\right)}{\left(\left(x_{1}-y_{1}\right)^{2}+\left(x_{2}-y_{2}\right)^{2}\right)^{3 / 2}}, \\
\dot{y_{1}}=\frac{\alpha \Gamma_{1}\left(y_{2}-x_{2}\right)}{\left(\left(x_{1}-x_{1}\right)^{2}+\left(x_{2}-y_{2}\right)^{2}\right)^{3 / 2}}, \\
\dot{y_{2}}=-\frac{\alpha \Gamma_{1}\left(y_{1}-x_{1}\right)}{\left(\left(x_{1}-x_{1}\right)^{2}+\left(x_{2}-y_{2}\right)^{2}\right)^{3 / 2}},
\end{array}\right.
$$

where a dot over a variable denotes the derivative with respect to time. Further setting $z_{1}=x_{1}+i x_{2}$ and $z_{2}=y_{1}+i y_{2}$, where $i$ is the imaginary unit, we have

$$
\left\{\begin{array}{c}
\dot{z}_{1}=-i \alpha \Gamma_{2} \frac{z_{1}-z_{2}}{\left|z_{1}-z_{2}\right|^{3 / 2}} \\
\dot{z}_{2}=-i \alpha \Gamma_{1} \frac{z_{2}-z_{1}}{\left|z_{1}-z_{2}\right|^{3 / 2}}
\end{array}\right.
$$

We see from direct calculation that when $\Gamma_{1}+\Gamma_{2} \neq 0$,

$$
C=\frac{\Gamma_{1} z_{1}+\Gamma_{2} z_{2}}{\Gamma_{1}+\Gamma_{2}}, \quad D=\left|z_{1}-z_{2}\right|,
$$

are conserved quantities. $C$ is known as the center of vorticity. Utilizing these quantities, the equations can be decoupled to obtain

$$
\left(\begin{array}{c}
\dot{z_{1}} \\
\dot{z_{2}}
\end{array}\right)=-\frac{i \alpha\left(\Gamma_{1}+\Gamma_{2}\right)}{D^{3 / 2}}\left(\begin{array}{ll}
1 & 0 \\
0 & 1
\end{array}\right)\left(\begin{array}{c}
z_{1}-C \\
z_{2}-C
\end{array}\right) .
$$

The above equation can be solved explicitly and we have

$$
z_{j}(t)=\left(z_{j}(0)-C\right) e^{i \omega t}+C
$$


for $j=1,2$, where $\omega=-\alpha\left(\Gamma_{1}+\Gamma_{2}\right) / D^{3 / 2}$. This shows that the two filaments rotate in a two-dimensional circular pattern and the center and radius of rotation is determined by the center of vorticity. When $\Gamma_{1}+\Gamma_{2}=0$, we see that $z_{1}-z_{2}$ is conserved and hence we have

$$
\dot{z}_{j}=-\frac{i \alpha \Gamma_{2}}{D^{3 / 2}} w_{0}=\text { const. }
$$

for $j=1,2$ with $w_{0}=z_{1}(0)-z_{2}(0)$. This shows that the two filaments travel in a straight line at a constant speed while keeping their parallel configuration. These dynamics of the filaments directly correspond to the motion of two point vortices moving in a plane, which is well known in the literature such as Newton [14]. Hence, we see that system (2.2) is capable of describing the motion of two parallel lines in the expected manner.

\section{Leapfrogging for a Pair of Filaments with Vorticity Strengths of the Same Sign}

We consider the case when the two filaments are arranged as coaxial circles and $\Gamma_{1}, \Gamma_{2}>0$. Rescaling the time variable by a factor of $\Gamma_{2}$ in (2.2) yields

$$
\left\{\begin{array}{c}
\boldsymbol{X}_{t}=\beta \frac{\boldsymbol{X}_{\xi} \times \boldsymbol{X}_{\xi \xi}}{\left|\boldsymbol{X}_{\xi}\right|^{3}}-\alpha \frac{\boldsymbol{Y}_{\xi} \times(\boldsymbol{X}-\boldsymbol{Y})}{|\boldsymbol{X}-\boldsymbol{Y}|^{3}} \\
\boldsymbol{Y}_{t}=\frac{\boldsymbol{Y}_{\xi} \times \boldsymbol{Y}_{\xi \xi}}{\left|\boldsymbol{Y}_{\xi}\right|^{3}}-\alpha \beta \frac{\boldsymbol{X}_{\xi} \times(\boldsymbol{Y}-\boldsymbol{X})}{|\boldsymbol{X}-\boldsymbol{Y}|^{3}}
\end{array}\right.
$$

where $\beta=\Gamma_{1} / \Gamma_{2}$. We assume without loss of generality that $\beta \geq 1$, since the case $\beta<1$ is reduced to the case $\beta>1$ by renaming the filaments.

Suppose that for some $R_{1,0}, R_{2,0}>0$ and $z_{1,0}, z_{2,0} \in \mathbf{R}$, the initial filaments $\boldsymbol{X}_{0}$ and $\boldsymbol{Y}_{0}$ are parametrized by $\xi \in[0,2 \pi)$ as follows.

$$
\boldsymbol{X}_{0}(\xi)={ }^{t}\left(R_{1,0} \cos (\xi), R_{1,0} \sin (\xi), z_{1,0}\right), \quad \boldsymbol{Y}_{0}(\xi)={ }^{t}\left(R_{2,0} \cos (\xi), R_{2,0} \sin (\xi), z_{2,0}\right),
$$

where we assume that $\left(R_{1,0}-R_{2,0}\right)^{2}+\left(z_{1,0}-z_{2,0}\right)^{2}>0$, which means that the two circles are not overlapping. Now, we make the ansatz

$\boldsymbol{X}(\xi, t)={ }^{t}\left(R_{1}(t) \cos (\xi), R_{1}(t) \sin (\xi), z_{1}(t)\right), \quad \boldsymbol{Y}(\xi, t)={ }^{t}\left(R_{2}(t) \cos (\xi), R_{2}(t) \sin (\xi), z_{2}(t)\right)$, 
and substitute it into (3.1). From the equation for $\boldsymbol{X}$ we have

$$
\begin{aligned}
\dot{R}_{1} \cos (\xi) & =-\frac{\alpha R_{2}\left(z_{1}-z_{2}\right) \cos (\xi)}{\left(\left(R_{1}-R_{2}\right)^{2}+\left(z_{1}-z_{2}\right)^{2}\right)^{3 / 2}}, \\
\dot{R}_{1} \sin (\xi) & =-\frac{\alpha R_{2}\left(z_{1}-z_{2}\right) \sin (\xi)}{\left(\left(R_{1}-R_{2}\right)^{2}+\left(z_{1}-z_{2}\right)^{2}\right)^{3 / 2}}, \\
\dot{z}_{1} & =\frac{\beta}{R_{1}}+\frac{\alpha R_{2}\left(R_{1}-R_{2}\right)}{\left(\left(R_{1}-R_{2}\right)^{2}+\left(z_{1}-z_{2}\right)^{2}\right)^{3 / 2}} .
\end{aligned}
$$

The dependence of the system on $\xi$ is eliminated by multiplying the first two equations by $\cos (\xi)$ and $\sin (\xi)$, respectively, and adding. The equations for $\boldsymbol{Y}$ are calculated in the same way and we arrive at

$$
\left\{\begin{array}{l}
\dot{R}_{1}=-\frac{\alpha R_{2}\left(z_{1}-z_{2}\right)}{\left(\left(R_{1}-R_{2}\right)^{2}+\left(z_{1}-z_{2}\right)^{2}\right)^{3 / 2}}, \\
\dot{z}_{1}=\frac{\beta}{R_{1}}+\frac{\alpha R_{2}\left(R_{1}-R_{2}\right)}{\left(\left(R_{1}-R_{2}\right)^{2}+\left(z_{1}-z_{2}\right)^{2}\right)^{3 / 2}}, \\
\dot{R}_{2}=\frac{\alpha \beta R_{1}\left(z_{1}-z_{2}\right)}{\left(\left(R_{1}-R_{2}\right)^{2}+\left(z_{1}-z_{2}\right)^{2}\right)^{3 / 2}}, \\
\dot{z}_{2}=\frac{1}{R_{2}}-\frac{\alpha \beta R_{1}\left(R_{1}-R_{2}\right)}{\left(\left(R_{1}-R_{2}\right)^{2}+\left(z_{1}-z_{2}\right)^{2}\right)^{3 / 2}}, \\
\left(R_{1}(0), z_{1}(0), R_{2}(0), z_{2}(0)\right)=\left(R_{1,0}, z_{1,0}, R_{2,0}, z_{2,0}\right) .
\end{array}\right.
$$

First, we observe that $z_{1}$ and $z_{2}$ can be reduced to one variable, namely $W=z_{1}-z_{2}$. Furthermore, we see by direct calculation that $\beta R_{1}^{2}+R_{2}^{2}$ is a conserved quantity. Hence, setting $d^{2}=\beta R_{1,0}^{2}+R_{2,0}^{2}$ with $d>0$, we make the change of variables

$$
R_{1}(t)=\frac{d}{\beta^{1 / 2}} \cos (\theta(t)), \quad R_{2}(t)=d \sin (\theta(t))
$$

to further reduce the system. We then arrive at

$$
\left\{\begin{array}{l}
\dot{\theta}=\frac{\alpha \beta^{1 / 2} W}{\left(\frac{d^{2}}{\beta}\left(\beta^{1 / 2} \sin \theta-\cos \theta\right)^{2}+W^{2}\right)^{3 / 2}}=: F_{1}(\theta, W), \\
\dot{W}=\frac{\beta^{3 / 2} \sin \theta-\cos \theta}{d \sin \theta \cos \theta}-\frac{\alpha d^{2}\left(\sin \theta+\beta^{1 / 2} \cos \theta\right)\left(\beta^{1 / 2} \sin \theta-\cos \theta\right)}{\beta^{1 / 2}\left(\frac{d^{2}}{\beta}\left(\beta^{1 / 2} \sin \theta-\cos \theta\right)^{2}+W^{2}\right)^{3 / 2}}=: F_{2}(\theta, W),
\end{array}\right.
$$


with initial data $\left(\theta_{0}, W_{0}\right)$. Here, $W_{0}=z_{1,0}-z_{2,0}$ and $\theta_{0}$ is determined uniquely from the relation

$$
R_{1,0}=\frac{d}{\beta^{1 / 2}} \cos \theta_{0}, \quad R_{2,0}=d \sin \theta_{0}
$$

Note that from our problem setting, $\left(\theta_{0}, W_{0}\right)$ is contained in the open set $\Omega_{\beta} \subset \mathbf{R}^{2}$ given by

$$
\Omega_{\beta}=\left\{(\theta, W) \in \mathbf{R}^{2} \mid 0<\theta<\frac{\pi}{2}, W \in \mathbf{R},(\theta, W) \neq\left(\theta_{\beta}, 0\right)\right\},
$$

where $\theta_{\beta}$ is the unique solution of

$$
\beta^{1 / 2} \sin \theta_{\beta}-\cos \theta_{\beta}=0,
$$

which is given explicitly by $\theta_{\beta}=\arctan \left(1 / \beta^{1 / 2}\right)$. The excluded point in the above definition corresponds to the two filaments overlapping. Since we can reconstruct the solution of (3.2) from the solution $(\theta(t), W(t))$ of $(3.3)$ by

$$
\begin{array}{r}
R_{1}(t)=\frac{d}{\beta^{1 / 2}} \cos (\theta(t)), \quad R_{2}(t)=d \sin (\theta(t)), \\
z_{1}(t)=\int_{0}^{t} \frac{\beta}{R_{1}(\tau)}+\frac{\alpha R_{2}(\tau)\left(R_{1}(\tau)-R_{2}(\tau)\right)}{\left(\left(R_{1}(\tau)-R_{2}(\tau)\right)^{2}+W(\tau)^{2}\right)^{3 / 2}} \mathrm{~d} \tau, \\
z_{2}(t)=\int_{0}^{t} \frac{1}{R_{2}(\tau)}-\frac{\alpha \beta R_{2}(\tau)\left(R_{1}(\tau)-R_{2}(\tau)\right)}{\left(\left(R_{1}(\tau)-R_{2}(\tau)\right)^{2}+W(\tau)^{2}\right)^{3 / 2}} \mathrm{~d} \tau,
\end{array}
$$

we focus on the solvability and behavior of the solution to system (3.3). It can be checked by direct calculation that the system (3.3) is a Hamiltonian system and the Hamiltonian $\mathcal{H}$ is given by

$$
\mathcal{H}(\theta, W)=\frac{1}{2 d} \log \left(\frac{(1-\sin \theta)^{\beta^{3 / 2}}(1-\cos \theta)}{(1+\sin \theta)^{\beta^{3 / 2}}(1+\cos \theta)}\right)-\frac{\alpha \beta^{1 / 2}}{\left(\frac{d^{2}}{\beta}\left(\beta^{1 / 2} \sin \theta-\cos \theta\right)^{2}+W^{2}\right)^{1 / 2}} .
$$

In other words, $F_{1}=\frac{\partial \mathcal{H}}{\partial W}$ and $F_{2}=-\frac{\partial \mathcal{H}}{\partial \theta}$. Of course, the Hamiltonian is a conserved quantity of motion. In this formulation, closed orbits revolving around the point $\left(\theta_{\beta}, 0\right)$ correspond to leapfrogging. From here, we treat (3.3) as a two-dimensional dynamical system in $\Omega_{\beta}$ with parameters $d, \beta$, and $\alpha$, and make use of many tools known for twodimensional dynamical systems and Hamiltonian systems, for example in Hirsch and Smale [10], to determine the dynamics of the filaments.

We state our main theorems. 
Theorem 3.1 For any $\alpha, d>0, \beta \geq 1$, and $\left(\theta_{0}, W_{0}\right) \in \Omega_{\beta}$, there exists a unique timeglobal solution $(\theta, W) \in C^{1}(\mathbf{R}) \times C^{1}(\mathbf{R})$ of $(3.3)$.

Theorem 3.2 In addition to the assumptions of Theorem 3.1, if we assume $0<\alpha<1 / 3$, then system (3.3) has two equilibrium points $\left(\theta_{*}, 0\right)$ and $\left(\theta_{* *}, 0\right)$ with $0<\theta_{*}<\theta_{\beta}$ and $\theta_{\beta}<\theta_{* *}<\pi / 2$, and the following two statements are equivalent.

(i) The solution with initial data $\left(\theta_{0}, W_{0}\right)$ is a leapfrogging solution. In other words, the solution curve is a closed orbit revolving around the point $\left(\theta_{\beta}, 0\right)$.

(ii) $\theta_{0} \in\left(\theta_{*}, \theta_{* *}\right)$ and $\mathcal{H}\left(\theta_{0}, W_{0}\right)<\min \left\{\mathcal{H}\left(\theta_{*}, 0\right), \mathcal{H}\left(\theta_{* *}, 0\right)\right\}$.

Remark 3.3 (Note on the assumption for $\alpha$ in Theorem 3.2) Recall that $\alpha>0$ was given by $\alpha=2 \delta / \log \left(\frac{L}{\varepsilon}\right)$, where $\delta, \varepsilon>0$ were small parameters with $L>0$ fixed. These parameters were introduced in the course of the derivation of the model system (2.2). Hence, it is natural to assume that $\alpha$ is small and also important that the smallness assumption for $\alpha$ in Theorem 3.2 is independent of the parameters $d$ and $\beta$. This allows us to treat different configurations of the filaments in the framework of one model, as opposed to models with different parameters depending on the configuration.

The rest of the section is devoted to the proof the above two theorems.

Proof of Theorem 3.1. Since $F_{1}$ and $F_{2}$ are smooth in $\Omega_{\beta}$, the time-local unique solvability is known. Suppose the maximum existence time $T>0$ is finite. From the standard theory of dynamical systems, for any compact set $K \subset \Omega_{\beta}$, there exists $t^{\prime} \in[0, T)$ such that $\left(\theta\left(t^{\prime}\right), W\left(t^{\prime}\right)\right) \notin K$. On the other hand, since the Hamiltonian is conserved, there exists $\eta>0$ and $r>0$ such that for all $t \in[0, T)$,

$$
(\theta(t), W(t)) \in\left(\left[\eta, \frac{\pi}{2}-\eta\right] \times \mathbf{R}\right) \backslash B_{r}\left(\theta_{\beta}, 0\right),
$$

where $B_{r}\left(\theta_{\beta}, 0\right)$ is the open ball with center $\left(\theta_{\beta}, 0\right)$ and radius $r$. This follows from the fact that the Hamiltonian diverges to $-\infty$ at $\theta=0, \pi / 2$ uniformly with respect to $W$ and at the point $\left(\theta_{\beta}, 0\right)$. In particular, since the solution curve is uniformly away from the point $\left(\theta_{\beta}, 0\right)$, there exists $c_{0}>0$ such that

$$
\frac{d^{2}}{\beta}\left(\beta^{1 / 2} \sin \theta(t)-\cos \theta(t)\right)^{2}+W(t)^{2} \geq c_{0}
$$

for all $t \in[0, T)$. Hence from the second equation in (3.3), we have

$$
|\dot{W}| \leq \frac{\beta^{3 / 2}+1}{d \sin \eta \cos (\pi / 2-\eta)}+\frac{\alpha d^{2}\left(\beta^{1 / 2}+1\right)^{2}}{\beta^{1 / 2} c_{0}^{3 / 2}}=: M
$$

which yields

$$
|W(t)| \leq|W(0)|+M t \leq\left|W_{0}\right|+M T
$$


for all $t \in[0, T)$. Finally, this shows that for all $t \in[0, T),(\theta(t), W(t))$ is contained in the compact set $K^{\prime}$ given by

$$
K^{\prime}=\left(\left[\eta, \frac{\pi}{2}-\eta\right] \times\left[-\left|W_{0}\right|-M T,\left|W_{0}\right|+M T\right]\right) \backslash B_{r}\left(\theta_{\beta}, 0\right),
$$

which is a contradiction. The same argument holds for $t<0$ and hence, the solution exists globally in time and is defined for all $t \in \mathbf{R}$.

Proof of Theorem 3.2. We divide the proof of Theorem 3.2 into subsections. First we prove that system (3.3) has exactly two equilibriums as stated in Theorem 3.2.

\subsection{Equilibriums of System (3.3)}

From the form of $F_{1}$, we see that an equilibrium can only exist on the line segment $(0, \pi / 2) \times\{0\}$, and thus, we set $f(\theta):=F_{2}(\theta, 0)$ and investigate the zeroes of $f$. First we consider the zeroes in the interval $\left(0, \theta_{\beta}\right)$. Keeping in mind that $\beta^{1 / 2} \sin \theta-\cos \theta<0$ in $\left(0, \theta_{\beta}\right)$, by a change of variable $\theta=\arctan x$ we have

$$
f(\arctan x)=\frac{\left(1+x^{2}\right)^{1 / 2} g_{\alpha}(x)}{d x\left(\beta^{1 / 2} x-1\right)^{2}},
$$

where $g_{\alpha}$ is given by

$$
g_{\alpha}(x)=\beta^{5 / 3} x^{3}-\beta(2 \beta+1) x^{2}+\beta^{1 / 2}(\beta+2) x-1+\alpha \beta\left(x^{2}+\beta^{1 / 2} x\right)
$$

for $x \in\left(0,1 / \beta^{1 / 2}\right)$. We further make the change of variable $y=\beta^{1 / 2} x$ for simplification and investigate the zeroes of the function $h_{\alpha}$ given by

$$
h_{\alpha}(y)=\beta y^{3}-(2 \beta+1) y^{2}+(\beta+2) y-1+\alpha\left(y^{2}+\beta y\right)
$$

in the interval $I_{1}=(0,1)$. We treat $h_{\alpha}$ as a perturbation of $h_{0}$ given by

$$
h_{0}(y)=\beta y^{3}-(2 \beta+1) y^{2}+(\beta+2) y-1,
$$

which is $h_{\alpha}$ with $\alpha=0$ and prove that $h_{\alpha}$ has exactly one zero in $I_{1}$. We see from direct calculation that $h_{0}$ has one local maximum and one local minimum at

$$
y_{1}=\frac{\beta+2}{3 \beta}, \quad y_{2}=1
$$

respectively, and

$$
h_{0}\left(y_{1}\right)=\frac{4}{27 \beta^{2}}(\beta-1)^{3}>0, \quad h_{0}\left(y_{2}\right)=0
$$

Since the zero at $y_{2}$ is singular, we cannot directly apply the method of perturbation to $h_{\alpha}$. Instead, we analyze the positions of the local extrema for small $\alpha>0$ to determine 
the number of zeroes of $h_{\alpha}$. First, we observe that the discriminant $\Delta$ of the quadratic equation $h_{\alpha}^{\prime}(y)=0$ is given by

$$
\Delta=4\left[(1-3 \alpha) \beta^{2}-2(1+2 \alpha) \beta+(\alpha-1)\right]=: 4 \phi(\beta) .
$$

$\phi(\beta)=0$ has two roots $\beta_{ \pm}$given by

$$
\beta_{-}=\frac{1+2 \alpha-\sqrt{3 \alpha\left(3-\alpha-\alpha^{2}\right)}}{1-3 \alpha}, \quad \beta_{+}=\frac{1+2 \alpha+\sqrt{3 \alpha\left(3-\alpha-\alpha^{2}\right)}}{1-3 \alpha}
$$

and under the assumption $0<\alpha<1 / 3$, we see that

$$
\phi(\beta)<0 \text { for } 1 \leq \beta<\beta_{+}, \quad \phi(\beta) \geq 0 \text { for } \beta_{+} \leq \beta,
$$

where we also used the fact that $\phi(1)=-\alpha(9-\alpha)<0$. This shows that when $1 \leq \beta<\beta_{+}$, $\Delta<0$ which implies $h_{\alpha}^{\prime}>0$ for $y \in(0,1)$. Since, $h_{\alpha}(0)=-1$ and $h_{\alpha}(1)=\alpha(1+\beta)>0$, there is exactly one zero in $I_{1}$.

When $\beta_{+} \leq \beta$, the roots $y_{ \pm}$of $h_{\alpha}^{\prime}(y)=0$ are given by

$$
y_{ \pm}=\frac{2 \beta+1-\alpha \pm \sqrt{\phi(\beta)}}{3 \beta}
$$

where $y_{-}$is the local maximum and $y_{+}$is the local minimum. Since $h_{\alpha}$ is a third order polynomial, it is sufficient to prove that $h_{\alpha}\left(y_{+}\right)>0$ to prove that $h_{\alpha}$ has exactly one root. We have

$$
\begin{aligned}
y_{+} \geq \frac{1}{3 \beta}(2 \beta+1-\alpha) & \geq \frac{1}{3 \beta}\left(\beta+2+\left(\beta_{+}-1\right)-\alpha\right) \\
& =\frac{1}{3 \beta}\left\{\beta+2+\frac{\alpha^{1 / 2}}{1-3 \alpha}\left[\left(\left(3\left(3-\alpha-\alpha^{2}\right)\right)^{1 / 2}+5 \alpha^{1 / 2}-(1-3 \alpha) \alpha^{1 / 2}\right]\right\}\right. \\
& \geq \frac{\beta+2}{3 \beta},
\end{aligned}
$$

which implies $h_{0}\left(y_{+}\right) \geq 0$. Finally, we have

$$
h_{\alpha}\left(y_{+}\right)=h_{0}\left(y_{+}\right)+\alpha\left(y_{+}^{2}+\beta y_{+}\right)>0
$$

which shows that $h_{\alpha}$ also has exactly one root when $\beta_{+} \leq \beta$. Hence we have proven that for any $\beta \geq 1$ and $0<\alpha<1 / 3, h_{\alpha}$ has exactly one zero $y_{*}$ in $I_{1}$ and $h_{\alpha}^{\prime}\left(y_{*}\right)>0$. Hence, $\theta_{*}=\arctan \left(y_{*} / \beta^{1 / 2}\right)$ is the desired zero of $f(\theta)$ in the interval $\left(0, \theta_{\beta}\right)$ and we see that $f^{\prime}\left(\theta_{*}\right)>0$. By a similar argument, we see that there exists a unique $\theta_{* *} \in\left(\theta_{\beta}, \pi / 2\right)$ such that $f\left(\theta_{* *}\right)=0$ and $f^{\prime}\left(\theta_{* *}\right)>0$. We note here that because $\theta_{*}$ and $\theta_{* *}$ are the only zeroes in the interval $\left(0, \theta_{\beta}\right)$ and $\left(\theta_{\beta}, \pi / 2\right)$ respectively, and $f^{\prime}\left(\theta_{*}\right), f^{\prime}\left(\theta_{* *}\right)>0$, we have the following property for $f(\theta)$.

$$
\begin{aligned}
& f(\theta)<0, \text { for } \theta \in\left(0, \theta_{*}\right) \cup\left(\theta_{* *}, \pi / 2\right), \\
& f(\theta)>0, \text { for } \theta \in\left(\theta_{*}, \theta_{\beta}\right) \cup\left(\theta_{\beta}, \theta_{* *}\right) .
\end{aligned}
$$




\subsection{Analysis for Solutions with Initial Data of the Form $\left(\theta_{0}, 0\right)$}

Since a leapfrogging solution corresponds to a closed orbit revolving around the point $\left(\theta_{\beta}, 0\right)$ in $\Omega_{\beta}$, a leapfrogging solution always crosses the lines $\left(0, \theta_{\beta}\right) \times\{0\}$ and $\left(\theta_{\beta}, \pi / 2\right) \times$ $\{0\}$ in $\Omega_{\beta}$. To this end, we first characterize the solutions with initial data of the form $\left(\theta_{0}, 0\right)$, and prove that the condition given in Theorem 3.2 is necessary and sufficient for leapfrogging to occur.

First we prove that (ii) implies (i). Set $H_{*}:=\min \left\{\mathcal{H}\left(\theta_{*}, 0\right), \mathcal{H}\left(\theta_{* *}, 0\right)\right\}$. Let $\theta_{0} \in$ $\left(\theta_{*}, \theta_{* *}\right)$ satisfy $\mathcal{H}\left(\theta_{0}, 0\right)<H_{*}$. To make the situation more concrete, we further assume that $\mathcal{H}\left(\theta_{*}, 0\right)>\mathcal{H}\left(\theta_{* *}, 0\right)$ and make a remark on the case $\mathcal{H}\left(\theta_{*}, 0\right) \leq \mathcal{H}\left(\theta_{* *}, 0\right)$ at the end. From (3.5) and the fact that $\frac{\partial \mathcal{H}}{\partial \theta}(\theta, 0)=-f(\theta)$, we have

$$
\begin{aligned}
& \frac{\partial \mathcal{H}}{\partial \theta}(\theta, 0)>0, \quad \text { for } \theta \in\left(0, \theta_{*}\right) \cup\left(\theta_{* *}, \pi / 2\right), \\
& \frac{\partial \mathcal{H}}{\partial \theta}(\theta, 0)<0, \quad \text { for } \theta \in\left(\theta_{*}, \theta_{\beta}\right) \cup\left(\theta_{\beta}, \theta_{* *}\right) .
\end{aligned}
$$

Moreover, since $\mathcal{H}\left(\theta_{*}, 0\right)>\mathcal{H}\left(\theta_{* *}, 0\right)$, and $\mathcal{H}(\theta, 0) \rightarrow-\infty$ monotonically as $\theta \rightarrow \theta_{\beta}-$, there exists a unique $\tilde{\theta} \in\left(\theta_{*}, \theta_{\beta}\right)$ such that $\mathcal{H}(\tilde{\theta}, 0)=H_{*}$. This implies that $\theta_{0} \in\left(\tilde{\theta}, \theta_{* *}\right) \backslash\left\{\theta_{\beta}\right\}$.

We assume that $\theta_{0} \in\left(\tilde{\theta}, \theta_{\beta}\right)$ since the arguments for the case $\theta_{0} \in\left(\theta_{\beta}, \theta_{* *}\right)$ is the same. We prove that the unique time-global solution $(\theta(t), W(t))$ starting from $\left(\theta_{0}, 0\right)$ obtained in Theorem 3.1, which is defined for $t \in \mathbf{R}$, is a closed orbit revolving around $\left(\theta_{\beta}, 0\right)$. First, we show that the solution is bounded. We observe that as a function of $W$, the Hamiltonian achieves a minimum at $W=0$ for each fixed $\theta$. Hence for all $W \in \mathbf{R}$, we have

$$
\begin{gathered}
\mathcal{H}(\tilde{\theta}, W) \geq \mathcal{H}(\tilde{\theta}, 0)=H_{*}>\mathcal{H}\left(\theta_{0}, 0\right), \\
\mathcal{H}\left(\theta_{* *}, W\right) \geq \mathcal{H}\left(\theta_{* *}, 0\right)=H_{*}>\mathcal{H}\left(\theta_{0}, 0\right) .
\end{gathered}
$$

The above and from the conservation and continuity of the Hamiltonian, there exists $\eta>0$ and $r>0$ such that

$$
(\theta(t), W(t)) \in\left(\left[\tilde{\theta}+\eta, \theta_{* *}-\eta\right] \times \mathbf{R}\right) \backslash B_{r}\left(\theta_{\beta}, 0\right),
$$

for all $t \in \mathbf{R}$. Furthermore, if we set

$$
\phi(\theta):=\frac{1}{2 d} \log \left(\frac{(1-\sin \theta)^{\beta^{3 / 2}}(1-\cos \theta)}{(1+\sin \theta)^{\beta^{3 / 2}}(1+\cos \theta)}\right),
$$

we see that as a function of $\theta, \mathcal{H}(\theta, W)$ converges to $\phi$ uniformly as $W \rightarrow \infty$. Since we have

$$
\phi^{\prime}(\theta)=-\frac{\left(\beta^{3 / 2} \sin \theta-\cos \theta\right)}{d \cos \theta \sin \theta}
$$


we see that $\phi$ achieves a maximum at $\theta=\arctan \left(1 / \beta^{3 / 2}\right)=$ : $\theta_{c}$ with $0<\theta_{c}<\theta_{\beta}$ and $\phi$ is monotone in the intervals $\left(0, \theta_{c}\right)$ and $\left(\theta_{c}, \pi / 2\right)$. If $0<\theta_{c} \leq \tilde{\theta}$, for $\varepsilon_{1}>0$ given by

$$
\varepsilon_{1}=\frac{\alpha \beta^{1 / 2}}{2\left\{\frac{d^{2}}{\beta}\left(\beta^{1 / 2} \sin \theta_{* *}-\cos \theta_{* *}\right)^{2}\right\}^{1 / 2}},
$$

there exists $W_{1}>0$ such that for all $\theta \in\left(\tilde{\theta}, \theta_{* *}\right)$, and $W>W_{1}$ we have

$$
\mathcal{H}(\theta, W)>\phi(\theta)-\varepsilon_{1}>\phi\left(\theta_{* *}\right)-2 \varepsilon_{1}=\mathcal{H}\left(\theta_{* *}, 0\right)=H_{*}>\mathcal{H}\left(\theta_{0}, 0\right)
$$

If $\tilde{\theta}<\theta_{c}<\theta_{\beta}$, choose $\theta^{\prime} \in\left\{\tilde{\theta}, \theta_{* *}\right\}$ so that $\phi\left(\theta^{\prime}\right)=\min \left\{\phi(\tilde{\theta}), \phi\left(\theta_{* *}\right)\right\}$. Then for $\varepsilon_{2}>0$ given by

$$
\varepsilon_{2}=\frac{\alpha \beta^{1 / 2}}{2\left\{\frac{d^{2}}{\beta}\left(\beta^{1 / 2} \sin \theta^{\prime}-\cos \theta^{\prime}\right)^{2}\right\}^{1 / 2}},
$$

there exists $W_{2}>0$ such that for all $\theta \in\left(\tilde{\theta}, \theta_{* *}\right)$ and $W>W_{2}$, we have

$$
\mathcal{H}(\theta, W)>\phi(\theta)-\varepsilon_{2}>\phi\left(\theta^{\prime}\right)-2 \varepsilon_{2}=\mathcal{H}\left(\theta^{\prime}, 0\right)=H_{*}>\mathcal{H}\left(\theta_{0}, 0\right) .
$$

In either case, we see that the value of the Hamiltonian on the segment $\left[\tilde{\theta}, \theta_{* *}\right] \times\left\{W_{*}\right\}$, where $W_{*}=\max \left\{W_{1}, W_{2}\right\}$, is strictly greater than $\mathcal{H}\left(\theta_{0}, 0\right)$ and hence the solution curve cannot cross this segment. Since the Hamiltonian is symmetric with respect to $W=0$, we finally see that

$$
(\theta(t), W(t)) \in\left(\left[\tilde{\theta}+\eta, \theta_{* *}-\eta\right] \times\left[-W_{*}, W_{*}\right]\right) \backslash B_{r}\left(\theta_{\beta}, 0\right)=: K_{*},
$$

for all $t \in \mathbf{R}$, and in particular, the solution is bounded.

Next we set

$$
L_{0}:=\left\{(\theta, W) \in \Omega_{\beta} \mid \mathcal{H}(\theta, W)=\mathcal{H}\left(\theta_{0}, 0\right)\right\} \cap K_{*} .
$$

As a closed subset of the compact set $K_{*}, L_{0}$ is a compact subset of $\Omega_{\beta}$. From the conservation of the Hamiltonian and the way we chose $\eta, r$, and $W_{*}$, we see that $L_{0}$ is also an invariant set and hence we have

$$
L_{\omega}\left(\theta_{0}, 0\right) \subset L_{0}
$$

where $L_{\omega}\left(\theta_{0}, 0\right)$ is the $\omega$-limit set of $\left(\theta_{0}, 0\right)$. Since $(\theta(t), W(t))$ is bounded for $t>0$, it converges along some series $\left\{t_{n}\right\}_{n=1}^{\infty}$ with $t_{n} \rightarrow \infty$ as $n \rightarrow \infty$, and in particular, $L_{\omega}\left(\theta_{0}, 0\right)$ is not empty. Since $L_{\omega}\left(\theta_{0}, 0\right)$ is a non-empty compact set and contains no equilibriums (recall that the equilibriums $\left(\theta_{*}, 0\right)$ and $\left(\theta_{* *}, 0\right)$ are outside the set $\left.L_{0}\right)$, it is a closed orbit by the Poincaré-Bendixson Theorem. Moreover, the point $\left(\theta_{\beta}, 0\right)$ is in the interior of this closed orbit, because if it is not, then the closed orbit would enclose an open subset of $\Omega_{\beta}$ in which an equilibrium must exist, which leads to a contradiction. This proves that 
$L_{\omega}\left(\theta_{0}, 0\right)$ is a closed orbit revolving around $\left(\theta_{\beta}, 0\right)$. Since $L_{\omega}\left(\theta_{0}, 0\right) \subset L_{0}$, there exists $\theta_{1} \in\left(\tilde{\theta}+\eta, \theta_{\beta}\right)$ and $\theta_{2} \in\left(\theta_{\beta}, \theta_{* *}-\eta\right)$ such that $\left(\theta_{1}, 0\right),\left(\theta_{2}, 0\right) \in L_{\omega}\left(\theta_{0}, 0\right)$. The values $\theta_{1}$ and $\theta_{2}$ satisfying this property are unique in their respective intervals because the Hamiltonian is monotone along the line segments $\left[\tilde{\theta}+\eta, \theta_{\beta}\right] \times\{0\}$ and $\left[\theta_{\beta}, \theta_{* *}-\eta\right] \times\{0\}$. This uniqueness implies that $\theta_{1}=\theta_{0}$, which proves that $L_{\omega}\left(\theta_{0}, 0\right)$ coincides with the orbit starting from $\left(\theta_{0}, 0\right)$.

In summary, we have proven that the orbit starting from $\left(\theta_{0}, 0\right)$ is a closed orbit revolving around $\left(\theta_{\beta}, 0\right)$ corresponding to a leapfrogging solution. We further have the characterization

$$
L_{\omega}\left(\theta_{0}, 0\right)=L_{0}
$$

which we prove by contradiction. Suppose there exists $(\bar{\theta}, \bar{W}) \in L_{0}$ such that $(\bar{\theta}, \bar{W}) \notin$ $L_{\omega}\left(\theta_{0}, 0\right)$. We first see that $\bar{W} \neq 0$, since $(\bar{\theta}, 0) \in L_{0}$ implies $\bar{\theta}=\theta_{1}$ or $\theta_{2}$, which contradicts $(\bar{\theta}, 0) \notin L_{\omega}\left(\theta_{0}, 0\right)$. Henceforth, we assume $\bar{W}>0$ since the proof for the other case is the same. Now, if $\bar{\theta} \in\left[\tilde{\theta}+\eta, \theta_{1}\right]$, we have

$$
\mathcal{H}(\bar{\theta}, \bar{W})>\mathcal{H}(\bar{\theta}, 0) \geq \mathcal{H}\left(\theta_{1}, 0\right)=\mathcal{H}\left(\theta_{0}, 0\right)
$$

from the monotonicity of $\mathcal{H}$ along the line $\{\bar{\theta}\} \times \mathbf{R}$ and the monotonicity along the line segment $\left[\bar{\theta}, \theta_{1}\right] \times\{0\}$, and this contradicts $(\bar{\theta}, \bar{W}) \in L_{0}$. The case $\bar{\theta} \in\left[\theta_{2}, \theta_{* *}-\eta\right]$ leads to a contradiction by the same argument. If $\bar{\theta} \in\left(\theta_{1}, \theta_{2}\right)$ and $(\bar{\theta}, \bar{W})$ is in the interior of the closed orbit $L_{\omega}\left(\theta_{0}, 0\right)$, there exists $\tilde{W}>\bar{W}$ such that $(\bar{\theta}, \tilde{W}) \in L_{\omega}\left(\theta_{0}, 0\right)$. Then we have

$$
\mathcal{H}(\bar{\theta}, \bar{W})<\mathcal{H}(\bar{\theta}, \tilde{W})=\mathcal{H}\left(\theta_{0}, 0\right),
$$

which contradicts $(\bar{\theta}, \bar{W}) \in L_{0}$. Similarly, if $(\bar{\theta}, \bar{W})$ is outside of the closed orbit, there exists $\tilde{W}<\bar{W}$ such that $(\bar{\theta}, \tilde{W}) \in L_{\omega}\left(\theta_{0}, 0\right)$. Again, this implies the estimate

$$
\mathcal{H}(\bar{\theta}, \bar{W})>\mathcal{H}(\bar{\theta}, \tilde{W})=\mathcal{H}\left(\theta_{0}, 0\right),
$$

which contradicts $(\bar{\theta}, \bar{W}) \in L_{0}$. Hence we have $L_{\omega}\left(\theta_{0}, 0\right)=L_{0}$. We can express $L_{0}$ as

$$
L_{0}=\left\{(\theta, W) \in \Omega_{\beta} \mid \mathcal{H}(\theta, W)=\mathcal{H}\left(\theta_{0}, 0\right)\right\} \cap M,
$$

with $M=\left[\theta_{*}, \theta_{* *}\right] \times \mathbf{R}$, because the value of the Hamiltonian on $M \backslash K_{*}$ is different from $\mathcal{H}\left(\theta_{0}, 0\right)$, and thus, replacing $K_{*}$ with $M$ does not add any points. This expression will be utilized to derive the necessary and sufficient condition for leapfrogging to occur for solutions with general initial data.

Finally, we make some remarks on the case $\mathcal{H}\left(\theta_{*}, 0\right) \ngtr \mathcal{H}\left(\theta_{* *}, 0\right)$. When $\mathcal{H}\left(\theta_{*}, 0\right)=$ $\mathcal{H}\left(\theta_{* *}, 0\right)$, the same proof holds with $\tilde{\theta}=\theta_{*}$. When $\mathcal{H}\left(\theta_{*}, 0\right)<\mathcal{H}\left(\theta_{* *}, 0\right)$, there is a unique $\hat{\theta} \in\left(\theta_{\beta}, \theta_{* *}\right)$ such that $\mathcal{H}(\hat{\theta}, 0)=H_{*}$. This $\hat{\theta}$ plays the same role as $\tilde{\theta}$, and the same arguments for the case $\mathcal{H}\left(\theta_{*}, 0\right)<\mathcal{H}\left(\theta_{* *}, 0\right)$ holds.

Next we prove that (i) implies (ii). Suppose that a solution starting from $\left(\theta_{0}, 0\right)$ is a leapfrogging solution. Since $\mathcal{H}\left(\theta_{*}, 0\right)$ and $\mathcal{H}\left(\theta_{* *}, 0\right)$ are the maximum value of $\mathcal{H}(\theta, 0)$ in 
their respective intervals $\left(0, \theta_{\beta}\right)$ and $\left(\theta_{\beta}, \pi / 2\right)$, in order for a solution curve to cross over the segments $\left(0, \theta_{\beta}\right) \times\{0\}$ and $\left(\theta_{\beta}, \pi / 2\right) \times\{0\}$, the value of the Hamiltonian on this solution curve must be less than or equal to the smaller of the two. In other words, $\mathcal{H}\left(\theta_{0}, 0\right) \leq H_{*}$ holds. If $\mathcal{H}\left(\theta_{0}, 0\right)=H_{*}$ holds, the only possible points at which the solution curve can cross the segments $\left(0, \theta_{\beta}\right) \times\{0\}$ and $\left(\theta_{\beta}, \pi / 2\right) \times\{0\}$ are at the equilibrium points. This would result in the solution converging to one of the equilibrium points, and is not a leapfrogging solution. Hence, for a leapfrogging solution, $\mathcal{H}\left(\theta_{0}, 0\right)<H_{*}$ holds.

Furthermore, $\left(\theta_{0}, 0\right)$ is not on the lines $\left\{\theta_{*}\right\} \times \mathbf{R}$ or $\left\{\theta_{* *}\right\} \times \mathbf{R}$ since the value of the Hamiltonian is greater than or equal to $H_{*}$ along these lines. Consequently, if $\theta_{0} \in$ $\left(0, \theta_{*}\right) \cup\left(\theta_{* *}, \pi / 2\right)$, the solution curve cannot cross over from one side of these lines to the other, which means that the solution is not a leapfrogging solution. This implies that $\theta_{0} \in\left(\theta_{*}, \theta_{* *}\right)$, and condition (ii) holds.

We summarize the conclusions of this subsection in the following lemma.

Lemma 3.4 For initial data of the form $\left(\theta_{0}, 0\right) \in \Omega_{\beta}$, we have the following.

(i) If $\theta_{0} \in\left(\theta_{*}, \theta_{* *}\right)$ and $\mathcal{H}\left(\theta_{0}, 0\right)<H_{*}$, then the solution starting from $\left(\theta_{0}, 0\right)$ is a leapfrogging solution. Moreover, the closed orbit $L_{\omega}\left(\theta_{0}, 0\right)$ can be expressed as

$$
L_{\omega}\left(\theta_{0}, 0\right)=\left\{(\theta, W) \in \Omega_{\beta} \mid \mathcal{H}(\theta, W)=\mathcal{H}\left(\theta_{0}, 0\right)\right\} \cap M,
$$

where $M=\left[\theta_{*}, \theta_{* *}\right] \times \mathbf{R}$.

(ii) Otherwise, the solution is not a leapfrogging solution.

\subsection{Remarks on Solutions with General Initial Data}

Let $\left(\theta_{0}, W_{0}\right) \in \Omega_{\beta}$ satisfy $\theta_{0} \in\left(\theta_{*}, \theta_{* *}\right)$ and $\mathcal{H}\left(\theta_{0}, W_{0}\right)<H_{*}$. Since $\mathcal{H}(\theta, 0)$ takes all values between $-\infty$ and $H_{*}$ on the set $\left(\theta_{*}, \theta_{\beta}\right) \cup\left(\theta_{\beta}, \theta_{* *}\right)$, there exists $\theta_{L F} \in\left(\theta_{*}, \theta_{\beta}\right) \cup\left(\theta_{\beta}, \theta_{* *}\right)$ such that $\mathcal{H}\left(\theta_{L F}, 0\right)=\mathcal{H}\left(\theta_{0}, W_{0}\right)$. Moreover, from Lemma 3.4, the orbit containing $\left(\theta_{L F}, 0\right)$ is a closed orbit corresponding to a leapfrogging solution. Since

$$
\left(\theta_{0}, W_{0}\right) \in\left\{(\theta, W) \in \Omega_{\beta} \mid \mathcal{H}(\theta, W)=\mathcal{H}\left(\theta_{L F}, 0\right)\right\} \cap M,
$$

Lemma 3.4 implies that $\left(\theta_{0}, W_{0}\right)$ is on the closed orbit containing $\left(\theta_{L F}, 0\right)$ and hence, the solution starting from $\left(\theta_{0}, W_{0}\right)$ is a leapfrogging solution.

On the other hand, suppose either $\mathcal{H}\left(\theta_{0}, W_{0}\right) \geq H_{*}$ or $\theta_{0} \notin\left(\theta_{*}, \theta_{* *}\right)$ holds. We prove that solution curves starting from these initial data are not leapfrogging solutions. If $\mathcal{H}\left(\theta_{0}, W_{0}\right) \geq H_{*}$, then the solution starting from $\left(\theta_{0}, W_{0}\right)$ is not a leapfrogging solution since the value of the Hamiltonian of a leapfrogging solution is strictly less than $H_{*}$ from Lemma 3.4. If $\theta_{0} \notin\left(\theta_{*}, \theta_{* *}\right)$ holds, we only need to consider the case when $\mathcal{H}\left(\theta_{0}, W_{0}\right)<$ $H_{*}$ also holds. Since $\mathcal{H}\left(\theta_{0}, W_{0}\right)<H_{*}, \theta_{0} \in\left(0, \theta_{*}\right) \cup\left(\theta_{* *}, \pi / 2\right)$ because the value of the Hamiltonian on the lines $\left\{\theta_{*}\right\} \times \mathbf{R}$ and $\left\{\theta_{* *}\right\} \times \mathbf{R}$ are greater than or equal to $H_{*}$. Furthermore, since the Hamiltonian is conserved, the solution curve starting from $\left(\theta_{0}, W_{0}\right)$ cannot cross over from one side of these lines to the other and hence, the solution is not a leapfrogging solution. This finishes the proof of Theorem 3.2. 


\section{Leapfrogging for a Pair of Filaments with Vorticity Strengths of Opposite Signs}

We consider the case when the two filaments are a pair of coaxial circles with vorticity strengths of opposite signs. This amounts to considering system (3.2) with $\beta<0$. Again, since the case $-1<\beta<0$ is reduced to the case $\beta \leq-1$ by renaming the filaments and rescaling the time variable, we assume $\beta \leq-1$ without loss of generality. Setting $\gamma=-\beta$, system (3.2) reads

$$
\left\{\begin{array}{l}
\dot{R}_{1}=-\frac{\alpha R_{2}\left(z_{1}-z_{2}\right)}{\left(\left(R_{1}-R_{2}\right)^{2}+\left(z_{1}-z_{2}\right)^{2}\right)^{3 / 2}}, \\
\dot{z}_{1}=-\frac{\gamma}{R_{1}}+\frac{\alpha R_{2}\left(R_{1}-R_{2}\right)}{\left(\left(R_{1}-R_{2}\right)^{2}+\left(z_{1}-z_{2}\right)^{2}\right)^{3 / 2}}, \\
\dot{R}_{2}=-\frac{\alpha \gamma R_{1}\left(z_{1}-z_{2}\right)}{\left(\left(R_{1}-R_{2}\right)^{2}+\left(z_{1}-z_{2}\right)^{2}\right)^{3 / 2}}, \\
\dot{z}_{2}=\frac{1}{R_{2}}+\frac{\alpha \gamma R_{1}\left(R_{1}-R_{2}\right)}{\left(\left(R_{1}-R_{2}\right)^{2}+\left(z_{1}-z_{2}\right)^{2}\right)^{3 / 2}}, \\
\left(R_{1}(0), z_{1}(0), R_{2}(0), z_{2}(0)\right)=\left(R_{1,0}, z_{1,0}, R_{2,0}, z_{2,0}\right)
\end{array}\right.
$$

with $\gamma \geq 1$. Note that by the nature of the leapfrogging motion, if a solution of (4.1) corresponds to leapfrogging, $R_{1}-R_{2}$ must change signs in a time-periodic pattern. We see from direct calculation that $\gamma R_{1}^{2}-R_{2}^{2}$ is a conserved quantity. This shows that $\left(R_{1}, R_{2}\right)$ lies on the set defined by $\gamma R_{1}^{2}-R_{2}^{2}=d$, where $d=\gamma R_{1,0}^{2}-R_{2,0}^{2}$, in the first quadrant of the $R_{1}-R_{2}$ plane. When $d<0$, this is a hyperbola which approaches the line $R_{2}=\gamma^{1 / 2} R_{1}$ from above and since $\gamma \geq 1, R_{2}>R_{1}$ on the hyperbola. Hence, a solution cannot correspond to leapfrogging in this case. If $d=0,\left(R_{1}, R_{2}\right)$ lies on the line $R_{2}=\gamma^{1 / 2} R_{1}$. When $\gamma>1$, $R_{2}>R_{1}$ and cannot correspond to leapfrogging. When $\gamma=1, R_{1}=R_{2}$ throughout the motion, and the two filaments approaching would result in the collision of the two filaments. This in itself is an interesting phenomenon, but is not a leapfrogging solution. When $d>0,\left(R_{1}, R_{2}\right)$ lies on a hyperbola which approaches the line $R_{2}=\gamma^{1 / 2} R_{1}$ from below. If $\gamma=1$, then $R_{1}>R_{2}$ on this hyperbola and leapfrogging cannot occur. If $\gamma>1$, there is a possibility that the solution of (4.1) is a leapfrogging solution, and we investigate in more detail.

First, we make the following change of variables.

$$
R_{1}(t)=\left(\frac{d}{\gamma}\right)^{1 / 2} \cosh (\theta(t)), \quad R_{2}(t)=d^{1 / 2} \sinh (\theta(t)), \quad W(t)=z_{1}(t)-z_{2}(t),
$$


which yields

$$
\left\{\begin{aligned}
\dot{\theta} & =-\frac{\alpha \gamma^{1 / 2} W}{\left(\frac{d}{\gamma}\left(\cosh \theta-\gamma^{1 / 2} \sinh \theta\right)^{2}+W^{2}\right)^{3 / 2}}=: G_{1}(\theta, W), \\
\dot{W} & =-\frac{1}{d^{1 / 2}}\left(\frac{\gamma^{3 / 2}}{\cosh \theta}+\frac{1}{\sinh \theta}\right)+\frac{\alpha d\left(\sinh \theta-\gamma^{1 / 2} \cosh \theta\right)\left(\cosh \theta-\gamma^{1 / 2} \sinh \theta\right)}{\gamma^{1 / 2}\left(\frac{d}{\gamma}\left(\cosh \theta-\gamma^{1 / 2} \sinh \theta\right)^{2}+W^{2}\right)^{3 / 2}} \\
& =: G_{2}(\theta, W),
\end{aligned}\right.
$$

with initial data $\left(\theta_{0}, W_{0}\right)$, which is determined by

$$
R_{1,0}=\left(\frac{d}{\gamma}\right)^{1 / 2} \cosh \left(\theta_{0}\right), \quad R_{2,0}=d^{1 / 2} \sinh \left(\theta_{0}\right), \quad W_{0}=z_{1,0}-z_{2,0}
$$

The phase space $\Omega_{\gamma} \subset \mathbf{R}^{2}$ is given by

$$
\Omega_{\gamma}=\left\{(\theta, W) \in \mathbf{R}^{2} \mid 0<\theta<\infty, W \in \mathbf{R},(\theta, W) \neq\left(\theta_{\gamma}, 0\right)\right\},
$$

where $\theta_{\gamma} \in(0, \infty)$ is the unique solution of

$$
\cosh \theta_{\gamma}-\gamma^{1 / 2} \sinh \theta_{\gamma}=0
$$

given explicitly by $\theta_{\gamma}=\operatorname{artanh}\left(1 / \gamma^{1 / 2}\right)$. The point $\left(\theta_{\gamma}, 0\right)$ corresponds to the two filaments overlapping. In this formulation, leapfrogging solutions correspond to closed orbits revolving around the point $\left(\theta_{\gamma}, 0\right)$. System (4.2) is of Hamiltonian form and the Hamiltonian $\mathcal{G}$ is given by

$$
\begin{gathered}
\mathcal{G}(\theta, W)=\frac{1}{d^{1 / 2}}\left(2 \gamma^{3 / 2} \arctan (\tanh (\theta / 2))+\log (\tanh (\theta / 2))\right) \\
+\frac{\alpha \gamma^{1 / 2}}{\left(\frac{d}{\gamma}\left(\cosh \theta-\gamma^{1 / 2} \sinh \theta\right)^{2}+W^{2}\right)^{1 / 2}} .
\end{gathered}
$$

We state our main theorems.

Theorem 4.1 For any $d>0, \alpha>0, \gamma>1$, and initial data $\left(\theta_{0}, W_{0}\right) \in \Omega_{\gamma}$, system (4.2) has a unique time-global solution $(\theta, W) \in C^{1}(\mathbf{R}) \times C^{1}(\mathbf{R})$.

Theorem 4.2 Let $0<\alpha<1 / 3$. There exists $\gamma_{*} \in(1, \infty)$ such that for any $d>0$, the following holds. When $1<\gamma \leq \gamma_{*}$, system (4.2) has no equilibriums and the following two statements are equivalent.

(i) The solution starting from $\left(\theta_{0}, W_{0}\right) \in \Omega_{\gamma}$ is a leapfrogging solution.

(ii) $\mathcal{G}\left(\theta_{0}, W_{0}\right)>\frac{\pi \gamma^{3 / 2}}{2 d^{1 / 2}}$. 
When $\gamma_{*}<\gamma$, system (4.2) has one equilibrium $\left(\theta_{*}, 0\right)$ with $\theta_{*} \in\left(\theta_{\gamma}, \infty\right)$, and the following two statements are equivalent.

(iii) The solution starting from $\left(\theta_{0}, W_{0}\right) \in \Omega_{\gamma}$ is a leapfrogging solution.

(iv) $\theta_{0} \in\left(\bar{\theta}, \theta_{*}\right)$ and $\mathcal{G}\left(\theta_{0}, W_{0}\right)>\mathcal{G}\left(\theta_{*}, 0\right)$.

Here, $\bar{\theta} \in\left(0, \theta_{\gamma}\right)$ is the unique value satisfying $\mathcal{G}(\bar{\theta}, 0)=\mathcal{G}\left(\theta_{*}, 0\right)$.

Proof of Theorem 4.1. The arguments of the proof are the same as that of Theorem 3.1 and hence, we only give the details for the essential parts. Let $\left(\theta_{0}, W_{0}\right) \in \Omega_{\gamma}$ and set $G_{0}:=\mathcal{G}\left(\theta_{0}, W_{0}\right)$. Since the unique existence of the time-local solution is known, we give an a priori estimate of the solution $(\theta(t), W(t))$ on the time interval $[0, T)$ to show that the solution is global in time. We first see that $\operatorname{since} \cosh \theta-\gamma^{1 / 2} \sinh \theta>0$ in $\left(0, \theta_{\gamma}\right)$,

$$
G_{2}(\theta, 0)=-\frac{1}{d^{1 / 2}}\left\{\frac{\gamma^{3 / 2} \sinh \theta+\cosh \theta}{\sinh \theta \cosh \theta}-\frac{\alpha \gamma\left(\sinh \theta-\gamma^{1 / 2} \cosh \theta\right)}{\left(\cosh \theta-\gamma^{1 / 2} \sinh \theta\right)^{2}}\right\}<0,
$$

which implies $\frac{\partial \mathcal{G}}{\partial \theta}(\theta, 0)>0$ in $\left(0, \theta_{\gamma}\right)$. By direct calculation, we see that $\mathcal{G}(\theta, 0) \rightarrow-\infty$ as $\theta \rightarrow 0$ and $\mathcal{G}(\theta, 0) \rightarrow \infty$ as $\theta \rightarrow \theta_{\gamma}$. This along with the monotonicity of $\mathcal{G}(\theta, 0)$ implies that there exists a unique $\theta_{1} \in\left(0, \theta_{\gamma}\right)$ such that $\mathcal{G}\left(\theta_{1}, 0\right)=G_{0} / 2$. Furthermore, $\mathcal{G}(\theta, W)$ is a strictly decreasing function of $|W|$ for each fixed $\theta$. Hence, the value of $\mathcal{G}$ is strictly less than $G_{0}$ along the line $\left\{\theta_{1}\right\} \times \mathbf{R}$. Then, the conservation of the Hamiltonian implies that $\theta_{1} \leq \theta(t)$ for any $t \in[0, T)$. Additionally, since $\mathcal{G}(\theta, W) \rightarrow \infty$ as $(\theta, W) \rightarrow\left(\theta_{\gamma}, 0\right)$, there exists $r>0$ such that

$$
(\theta(t), W(t)) \in\left(\left[\theta_{1}, \infty\right) \times \mathbf{R}\right) \backslash B_{r}\left(\theta_{\gamma}, 0\right)
$$

for all $t \in[0, T)$. This implies that for some $c_{0}>0$,

$$
\frac{d}{\gamma}\left(\cosh \theta(t)-\gamma^{1 / 2} \sinh \theta(t)\right)^{2}+W(t)^{2} \geq c_{0}
$$

for all $t \in[0, T)$. Hence we have

$$
|\dot{W}| \leq \frac{1}{d^{1 / 2}}\left(\gamma^{3 / 2}+\frac{1}{\sinh \theta_{1}}\right)+\frac{\alpha d\left|\sinh \theta-\gamma^{1 / 2} \cosh \theta\right|\left|\cosh \theta-\gamma^{1 / 2} \sinh \theta\right|}{\gamma^{1 / 2}\left(\frac{d}{\gamma}\left(\cosh \theta-\gamma^{1 / 2} \sinh \theta\right)^{2}+W^{2}\right)^{3 / 2}}
$$

Note that the second term is bounded regardless of the size of $\theta$. Indeed, for $0 \leq \theta \leq M$ with $\theta_{\gamma}<M$, we have

$$
\begin{aligned}
& \frac{\alpha d\left|\sinh \theta-\gamma^{1 / 2} \cosh \theta\right|\left|\cosh \theta-\gamma^{1 / 2} \sinh \theta\right|}{\gamma^{1 / 2}\left(\frac{d}{\gamma}\left(\cosh \theta-\gamma^{1 / 2} \sinh \theta\right)^{2}+W^{2}\right)^{3 / 2}} \\
& \quad \leq \frac{\alpha d\left(\sinh M+\gamma^{1 / 2} \cosh M\right)\left(\cosh M+\gamma^{1 / 2} \sinh M\right)}{\gamma^{1 / 2} c_{0}^{3 / 2}}=: M_{1} .
\end{aligned}
$$


For $M<\theta$, we have

$$
\begin{aligned}
& \frac{\alpha d\left|\sinh \theta-\gamma^{1 / 2} \cosh \theta\right|\left|\cosh \theta-\gamma^{1 / 2} \sinh \theta\right|}{\gamma^{1 / 2}\left(\frac{d}{\gamma}\left(\cosh \theta-\gamma^{1 / 2} \sinh \theta\right)^{2}+W^{2}\right)^{3 / 2}} \\
& \quad \leq \frac{\alpha \gamma^{1 / 2}\left|\sinh \theta-\gamma^{1 / 2} \cosh \theta\right|\left|\cosh \theta-\gamma^{1 / 2} \sinh \theta\right|}{d^{1 / 2}\left|\cosh \theta-\gamma^{1 / 2} \sinh \theta\right|^{3}} \\
& \quad \leq \frac{2 \alpha \gamma^{1 / 2}\left|\left(1-\gamma^{1 / 2}\right) e^{\theta}-\left(1+\gamma^{1 / 2}\right) e^{-\theta}\right|\left|\left(1-\gamma^{1 / 2}\right) e^{\theta}+\left(1+\gamma^{1 / 2}\right) e^{-\theta}\right|}{d^{1 / 2}\left(\left(\gamma^{1 / 2}-1\right) e^{\theta}-\left(1+\gamma^{1 / 2}\right) e^{-\theta}\right)^{3}} \\
& \quad=\frac{2 \alpha \gamma^{1 / 2} e^{-\theta}\left|\left(1-\gamma^{1 / 2}\right)-\left(1+\gamma^{1 / 2}\right) e^{-2 \theta}\right|\left|\left(1-\gamma^{1 / 2}\right)+\left(1+\gamma^{1 / 2}\right) e^{-2 \theta}\right|}{d^{1 / 2}\left(\left(\gamma^{1 / 2}-1\right)-\left(1+\gamma^{1 / 2}\right) e^{-2 \theta}\right)^{3}} \\
& \leq \frac{4 \alpha \gamma^{1 / 2} e^{-M}\left(\left(\gamma^{1 / 2}-1\right)+\left(1+\gamma^{1 / 2}\right) e^{-2 M}\right)^{2}}{d^{1 / 2}\left(\left(\gamma^{1 / 2}-1\right)-\left(1+\gamma^{1 / 2}\right) e^{-2 M}\right)^{3}}=: M_{2} .
\end{aligned}
$$

Hence we have

$$
|\dot{W}| \leq \frac{1}{d^{1 / 2}}\left(\gamma^{3 / 2}+\frac{1}{\sinh \theta_{1}}\right)+\max \left\{M_{1}, M_{2}\right\}=: M_{0},
$$

which yields

$$
|W(t)| \leq\left|W_{0}\right|+M T
$$

for all $t \in[0, T)$. This further yields

$$
|\dot{\theta}| \leq \frac{\alpha \gamma^{1 / 2}\left(\left|W_{0}\right|+M T\right)}{c_{0}^{3 / 2}}=: C_{0},
$$

which implies

$$
|\theta(t)| \leq\left|\theta_{0}\right|+C_{0} T
$$

for all $t \in[0, T)$, and these estimates for $\theta(t)$ and $W(t)$ are the desired a priori estimates.

Proof of Theorem 4.2. We first prove the existence of $\gamma_{*} \in(1, \infty)$ as stated in the theorem.

\subsection{The Existence of $\gamma_{*} \in(1, \infty)$}

From the form of system (4.2), an equilibrium can only exist on the line $W=0$, and hence we look for zeroes of $g(\theta):=G_{2}(\theta, 0)$. We have already seen that $g(\theta)<0$ in $\left(0, \theta_{\gamma}\right)$, so we consider $g(\theta)$ in the interval $\left(\theta_{\gamma}, \infty\right)$. By the change of variable $\theta=\operatorname{artanh} x$, we have

$$
g(\operatorname{artanh} x)=-\frac{(1+x)^{1 / 2}(1-x)^{1 / 2}}{d^{1 / 2} x\left(1-\gamma^{1 / 2} x\right)^{2}} g_{\alpha}(x),
$$


where $x \in\left(1 / \gamma^{1 / 2}, 1\right)$ and $g_{\alpha}$ is given by

$$
g_{\alpha}(x)=\gamma^{5 / 2} x^{3}+(1-2 \gamma) \gamma x^{2}+(\gamma-2) \gamma^{1 / 2} x+1+\alpha \gamma^{1 / 2} x\left(\gamma^{1 / 2} x-\gamma^{3 / 2}\right) .
$$

We further set $y=\gamma^{1 / 2} x$ to simplify and obtain

$$
h_{\alpha}(y)=\gamma y^{3}+(1-2 \gamma) y^{2}+(\gamma-2) y+1+\alpha y(y-\gamma)
$$

for $y \in\left(1, \gamma^{1 / 2}\right)$. We see by direct calculation that $h_{\alpha}$ takes local maximum at $y_{-}$and local minimum at $y_{+}$, each given by

$$
y_{ \pm}=\frac{-(1-2 \gamma+\alpha) \pm \sqrt{(\gamma+1)^{2}+\alpha\left(3 \gamma^{2}-4 \gamma+2+\alpha\right)}}{3 \gamma}
$$

Note that $3 \gamma^{2}-4 \gamma+2+\alpha>0$ for all $\gamma>1$ and in particular, $y_{+}-y_{-} \geq 2 / 3$ for all $\gamma>1$ and $\alpha>0$. We further have

$$
y_{-}<\frac{-1+2 \gamma-\alpha}{3 \gamma}=\frac{2}{3}-\frac{(1+\alpha)}{3 \gamma}<1
$$

Hence, regardless of the exact value of $y_{+}$, it is sufficient to evaluate the value of $h_{\alpha}$ at $y=1, \gamma^{1 / 2}$ to determine the behavior of $h_{\alpha}$ in the interval $\left(1, \gamma^{1 / 2}\right)$. We have

$$
h_{\alpha}(1)=\alpha(1-\gamma)<0
$$

and

$$
h_{\alpha}\left(\gamma^{1 / 2}\right)=\left(\gamma^{1 / 2}-1\right)^{2}\left(\gamma^{3 / 2}+1\right)+\alpha \gamma\left(1-\gamma^{1 / 2}\right)
$$

Setting $\eta:=\gamma^{1 / 2}$, we have

$$
h_{\alpha}(\eta)=(\eta-1)\left(\eta^{4}-3 \eta^{3}-\alpha \eta^{2}+\eta-1\right)=:(\eta-1) \phi(\eta) .
$$

After some differentiations, we can conclude that $\phi$ is monotonically increasing in $\eta>1$, and

$$
\phi(1)=-\alpha, \quad \phi(\eta) \rightarrow \infty(\eta \rightarrow \infty)
$$

which implies that there exists a unique $\eta_{*} \in(1, \infty)$ such that $\phi\left(\eta_{*}\right)=0$. Setting $\gamma_{*}=\eta_{*}^{2}$, we see that if $1<\gamma \leq \gamma_{*}, h_{\alpha}\left(\gamma^{1 / 2}\right) \leq 0$ and hence $h_{\alpha}<0$ in $\left(1, \gamma^{1 / 2}\right)$. This implies that $g_{\alpha}<0$ in $\left(\theta_{\gamma}, \infty\right)$ and hence, $g>0$ in $\left(\theta_{\gamma}, \infty\right)$. This shows that there are no equilibriums in this case.

If $\gamma_{*}<\gamma, h_{\alpha}\left(\gamma^{1 / 2}\right)>0$ which implies that there exists a unique $y_{*} \in\left(1, \gamma^{1 / 2}\right)$ such that $h_{\alpha}\left(y_{*}\right)=0$, and $h_{\alpha}<0$ in $\left(1, y_{*}\right)$ and $h_{\alpha}>0$ in $\left(y_{*}, \gamma^{1 / 2}\right)$. Then, setting $\theta_{*}=$ $\operatorname{artanh}\left(y_{*} / \gamma^{1 / 2}\right),\left(\theta_{*}, 0\right)$ is the unique equilibrium. 
The above arguments give the following profile for the Hamiltonian. When $1<\gamma \leq \gamma_{*}$,

$$
\left\{\begin{array}{l}
\frac{\partial \mathcal{G}}{\partial \theta}(\theta, 0)>0, \quad \theta \in\left(0, \theta_{\gamma}\right), \\
\frac{\partial \mathcal{G}}{\partial \theta}(\theta, 0)<0, \quad \theta \in\left(\theta_{\gamma}, \infty\right) .
\end{array}\right.
$$

When $\gamma_{*}<\gamma$,

$$
\left\{\begin{array}{l}
\frac{\partial \mathcal{G}}{\partial \theta}(\theta, 0)>0, \quad \theta \in\left(0, \theta_{\gamma}\right) \cup\left(\theta_{*}, \infty\right), \\
\frac{\partial \mathcal{G}}{\partial \theta}(\theta, 0)<0, \quad \theta \in\left(\theta_{\gamma}, \theta_{*}\right) .
\end{array}\right.
$$

We also see from direct calculation that

$$
\mathcal{G}(\theta, 0) \rightarrow-\infty(\theta \rightarrow 0), \quad \mathcal{G}(\theta, 0) \rightarrow \infty\left(\theta \rightarrow \theta_{\gamma}\right) .
$$

These properties will be used later.

Now we divide the proof of Theorem 4.2 into the cases $1<\gamma \leq \gamma_{*}$ and $\gamma_{*}<\gamma$. Like in Section 3, we determine the behavior of the solutions starting from an initial data of the form $\left(\theta_{0}, 0\right)$. The arguments for solutions starting from a general initial data are similar to that given in Section 3 and we will only give a brief remark on the matter at the end.

\subsection{The Case $1<\gamma \leq \gamma_{*}$}

We prove that condition (i) implies (ii) and vice versa. Suppose we have a leapfrogging solution, which in other words, is a solution for which the solution curve is a closed orbit revolving around the point $\left(\theta_{\gamma}, 0\right)$ in the phase space $\Omega_{\gamma}$. For this to happen, the orbit must cross over the line $\left(\theta_{\gamma}, \infty\right) \times\{0\}$. Since

$$
\mathcal{G}(\theta, 0) \rightarrow \frac{\pi \gamma^{3 / 2}}{2 d^{1 / 2}}
$$

as $\theta \rightarrow \infty$, the second property in (4.3) and the conservation of the Hamiltonian asserts that $\mathcal{G}\left(\theta_{0}, 0\right)>\frac{\pi \gamma^{3 / 2}}{2 d^{1 / 2}}$ must hold in order for the solution curve to cross over the line $\left(\theta_{\gamma}, \infty\right) \times\{0\}$.

Now, suppose $G_{0}:=\mathcal{G}\left(\theta_{0}, 0\right)>\frac{\pi \gamma^{3 / 2}}{2 d^{1 / 2}}$ and consider the solution $(\theta(t), W(t))$ starting from $\left(\theta_{0}, 0\right)$. First there exists $\theta_{1} \in\left(0, \theta_{\gamma}\right)$ and $\theta_{2} \in\left(\theta_{\gamma}, \infty\right)$, both unique in their respective intervals, such that

$$
\mathcal{G}\left(\theta_{1}, 0\right)=\mathcal{G}\left(\theta_{2}, 0\right)=G_{0} .
$$

Note that either $\theta_{1}$ or $\theta_{2}$ is $\theta_{0}$. Set $\zeta:=\theta_{1} / 2$. From the conservation of the Hamiltonian, there exists $r>0$ such that

$$
(\theta(t), W(t)) \in\left(\left[\theta_{1}-\zeta, \theta_{2}+\zeta\right] \times \mathbf{R}\right) \backslash B_{r}\left(\theta_{\gamma}, 0\right)
$$


for all $t \in \mathbf{R}$. Furthermore, if we set

$$
\psi(\theta):=\frac{1}{d^{1 / 2}}\left\{2 \gamma^{3 / 2} \arctan (\tanh (\theta / 2))+\log (\tanh (\theta / 2))\right\},
$$

we see that $\mathcal{G}(\theta, W)$ converges to $\psi(\theta)$ uniformly as $|W| \rightarrow \infty$ and this convergence is monotonically decreasing. Since we also have $\psi^{\prime}>0$ in $(0, \infty)$, for $\varepsilon_{1}=\frac{1}{2}\left(G_{0}-\psi\left(\theta_{2}+\zeta\right)\right)$ there exists $W_{*}>0$ such that for all $\theta \in\left[\theta_{1}-\zeta, \theta_{2}+\zeta\right]$ and $W$ satisfying $|W|>W_{*}$, we have

$$
\mathcal{G}(\theta, W)<\psi(\theta)+\varepsilon_{1}=\psi(\theta)+\frac{1}{2}\left(G_{0}-\psi\left(\theta_{2}+\zeta\right)\right)<G_{0}
$$

Again, the conservation of the Hamiltonian implies that

$$
(\theta(t), W(t)) \in\left(\left[\theta_{1}-\zeta, \theta_{2}+\zeta\right] \times\left[-W_{*}, W_{*}\right]\right) \backslash B_{r}\left(\theta_{\gamma}, 0\right)=: K .
$$

From here, the proof is the same as that of Section 3. The set $L_{1} \subset \Omega_{\gamma}$ given by

$$
L_{1}:=\left\{(\theta, W) \in \Omega_{\gamma} \mid \mathcal{G}(\theta, W)=G_{0}\right\} \cap K
$$

is a compact invariant set which shows that $L_{\omega}\left(\theta_{0}, 0\right)$, the $\omega$-limit set of $\left(\theta_{0}, 0\right)$, satisfies $L_{\omega}\left(\theta_{0}, 0\right) \subset L_{1}$. Moreover, since $L_{\omega}\left(\theta_{0}, 0\right)$ is non-empty, compact, and contains no equilibriums, it is a closed orbit enclosing the point $\left(\theta_{\gamma}, 0\right)$. It follows that $L_{1}=L_{\omega}\left(\theta_{0}, 0\right)$ and $L_{\omega}\left(\theta_{0}, 0\right)$ coincides with the solution curve starting from $\left(\theta_{0}, 0\right)$. Hence, the solution starting from $\left(\theta_{0}, 0\right)$ is a leapfrogging solution.

\subsection{The Case $\gamma_{*}<\gamma$}

We prove that (iii) implies (iv) and vice versa. From (4.4), we see that $\mathcal{G}(\theta, 0)$ takes its minimum value at $\theta=\theta_{*}$ in the interval $\left(\theta_{\gamma}, \infty\right)$. We also see from (4.4) and (4.5) that there is a unique $\bar{\theta} \in\left(0, \theta_{\gamma}\right)$ such that

$$
\mathcal{G}(\bar{\theta}, 0)=\mathcal{G}\left(\theta_{*}, 0\right)
$$

Suppose the solution starting from $\left(\theta_{0}, 0\right)$ is a leapfrogging solution. Since a leapfrogging solution must cross over the line $\left(\theta_{\gamma}, \infty\right) \times\{0\}$, the conservation of the Hamiltonian implies that $\mathcal{G}\left(\theta_{0}, 0\right)>\mathcal{G}\left(\theta_{*}, 0\right)$. This further implies that $\bar{\theta}<\theta_{0}$ because $\mathcal{G}(\theta, 0)<\mathcal{G}\left(\theta_{0}, 0\right)$ for any $\theta \in(0, \bar{\theta})$. If $\theta_{*}<\theta_{0}$, the solution curve starting from $\left(\theta_{0}, 0\right)$ cannot cross over the line $\left\{\theta_{*}\right\} \times \mathbf{R}$, and in turn is not a leapfrogging solution. This shows that $\theta_{0}<\theta_{*}$ must hold and hence condition (iv) is satisfied.

Now, suppose condition (iv) holds for $\left(\theta_{0}, 0\right) \in \Omega_{\gamma}$, and consider the solution $(\theta(t), W(t))$ starting from $\left(\theta_{0}, 0\right)$. The conservation of the Hamiltonian asserts that there exists $r>0$ such that

$$
(\theta(t), W(t)) \in\left(\left[\bar{\theta}-\zeta, \theta_{*}+\zeta\right] \times \mathbf{R}\right) \backslash B_{r}\left(\theta_{\gamma}, 0\right)
$$


for all $t \in \mathbf{R}$, where $\zeta=\frac{1}{2} \min \left\{\theta_{0}-\bar{\theta}, \theta_{*}-\theta_{0}\right\}$. Again, from the monotone and uniform convergence of $\mathcal{G}(\theta, W)$ as $|W| \rightarrow \infty$, we see that there exists $W_{*}>0$ such that

$$
(\theta(t), W(t)) \in\left(\left[\bar{\theta}-\zeta, \theta_{*}+\zeta\right] \times\left[-W_{*}, W_{*}\right]\right) \backslash B_{r}\left(\theta_{\gamma}, 0\right)
$$

for all $t \in \mathbf{R}$. The rest is the same as the previous case and we have the conclusion that the $\omega$-limit set $L_{\omega}\left(\theta_{0}, 0\right)$ of $\left(\theta_{0}, 0\right)$ is the desired closed orbit corresponding to a leapfrogging solution.

\subsection{Remarks on Solutions with General Initial Data}

We give a brief remark for solutions starting from general initial data $\left(\theta_{0}, W_{0}\right) \in \Omega_{\gamma}$. From the proof given so far, we see that in the case $1<\gamma \leq \gamma_{*}$, the collection of all closed orbits coincides with the collection of sets $\{L(G)\}$ where the set $L(G)$ is given by

$$
L(G):=\left\{(\theta, W) \in \Omega_{\gamma} \mid \mathcal{G}(\theta, W)=G\right\}
$$

for $G>\frac{\pi \gamma^{3 / 2}}{2 d^{1 / 2}}$. For a solution starting from $\left(\theta_{0}, W_{0}\right)$ to be a leapfrogging solution, it is necessary and sufficient that $\left(\theta_{0}, W_{0}\right) \in L(G)$ for some $G>\frac{\pi \gamma^{3 / 2}}{2 d^{1 / 2}}$. Similarly, in the case $\gamma_{*}<\gamma$, the collection of all closed orbits coincides with the collection of sets $\{K(G)\}$, where the set $K(G)$ is given by

$$
K(G):=\left(\left[\bar{\theta}, \theta_{*}\right] \times \mathbf{R}\right) \cap L(G)
$$

for $G>\mathcal{G}\left(\theta_{*}, 0\right)$. Again, for the solution to be a leapfrogging solution, it is necessary and sufficient that $\left(\theta_{0}, W_{0}\right) \in K(G)$ for some $G>\mathcal{G}\left(\theta_{*}, 0\right)$. In both cases, the conditions given

in Theorem 4.2 is a reinterpretation of these facts. This finishes the proof of Theorem 4.2 .

\section{Discussions and Conclusions}

We make some comparisons with existing models and make concluding remarks.

\subsection{Comparison with Existing Results}

We make a comparison between the results by Borisov, Kilin, and Mamev [2]. In [2], they consider the following model system.

$$
\left\{\begin{array}{l}
\dot{R}_{i}=-\frac{1}{R_{i}} \frac{\partial}{\partial Z_{i}} \sum_{j \neq i} \Gamma_{j} G\left(R_{i}, Z_{i}, R_{j}, Z_{j}\right) \\
\dot{Z}_{i}=\frac{1}{R_{i}} \frac{\partial}{\partial R_{i}}\left(\sum_{j \neq i} \Gamma_{j} G\left(R_{i}, Z_{i}, R_{j}, Z_{j}\right)\right)+\frac{\Gamma_{i}}{4 \pi R_{i}}\left(\log \frac{8 R_{i}}{a_{i}}-\frac{3}{4}\right),
\end{array}\right.
$$


where $i=1,2$ is the index for the two rings, $R_{i}$ are the radii of the rings, $Z_{i}$ are the distances along the common axis of symmetry, $\Gamma_{i}$ are the vorticity strengths of the rings, $a_{i}$ are the radii of the cross-section of the cores, which is taken to be a constant, and $G$ is given by

$$
G(z, r, \tilde{z}, \tilde{r})=\frac{(r \tilde{r})^{1 / 2}}{2 \pi}\left(\left(\frac{2}{k}-k\right) K(k)-\frac{2}{k} E(k)\right), \quad k=\left(\frac{4 r \tilde{r}}{(z-\tilde{z})^{2}+(r+\tilde{r})^{2}}\right)^{1 / 2} .
$$

$K(k)$ and $E(k)$ are the complete elliptic integrals of the first and second kind given by

$$
K(k)=\int_{0}^{1} \frac{\mathrm{d} x}{\sqrt{1-x^{2}} \sqrt{1-k^{2} x^{2}}}, \quad E(k)=\int_{0}^{1} \frac{\sqrt{1-k^{2} x^{2}}}{\sqrt{1-x^{2}}} \mathrm{~d} x .
$$

In [2], they analyze (5.1) to determine all the possible types of motion. The model used in [2] is derived as a system of ordinary differential equations (ODE), in other words, they only consider thin vortex rings as opposed to filaments with general shapes. The advantages of (5.1) over our model (2.2) is that it can incorporate the effect of the change in shape of the core and the effect of the change in vorticity distribution inside the core. Indeed, the second term on the right-hand side of the equation for $Z_{i}$ is written in a more general form given by

$$
\frac{\Gamma_{i}}{4 \pi R_{i}}\left(\log \frac{8 R_{i}}{a_{i}}-\frac{1}{2}+\Delta\left(a_{i}\right)\right)
$$

where

$$
\Delta\left(a_{i}\right)=\frac{1}{\Gamma_{i}^{2}} \int_{0}^{a_{i}} \frac{\gamma_{i}(s)^{2}}{s} \mathrm{~d} s, \quad \gamma_{i}(s)=2 \pi \int_{0}^{s} \omega_{i}(r) r \mathrm{~d} r .
$$

Here, $\gamma_{i}$ is the velocity circulation around the central part of the core and $\omega_{i}$ is the vorticity distribution of the cross-section of the core. The model considered in [2] corresponds to vortex rings with a circular core cross-section with constant radius and a uniform vorticity distribution. As was stated in the introduction, the ODE model has a long history behind its derivation and analysis, and is widely accepted as the model which describes the motion of interacting coaxial vortex rings.

On the other hand, we derived the model system (2.2) as a system of partial differential equations (PDE). The leapfrogging solutions obtained in Sections 3 and 4 are solutions of this PDE model, and hence it is possible to consider the stability (or the lack there of) of these solutions under non-symmetric perturbations, i.e. a perturbation in which the shape of the filament is deviated from a circle. This is not possible in the framework of the ODE model.

By comparing the systems (5.1) and (3.2), one can say that (5.1) is more focused on the precise description of the motion of the rings. In fact, system (3.2) can be seen as a simplification of the ODE model. This is especially apparent for the term describing the self-induced velocity in the equations for $z_{i}$ and $Z_{i}$. This is expected since our model 
neglects the effects of the finite core size. To this end, we give a numerical plot of the phase portraits of the system (3.3) to observe the possible dynamics of the circular filament pair described by our model. We do this to show that although our model is simpler, it is still able to capture the essential characteristics of the possible motions of vortex rings. The following plots were obtained by Mathematica as the level-sets of the Hamiltonian $\mathcal{H}(\theta, W)$. The parameters were set at $d=1, \alpha=0.1$, and plots are given for $\beta=1,2$, and 4 .

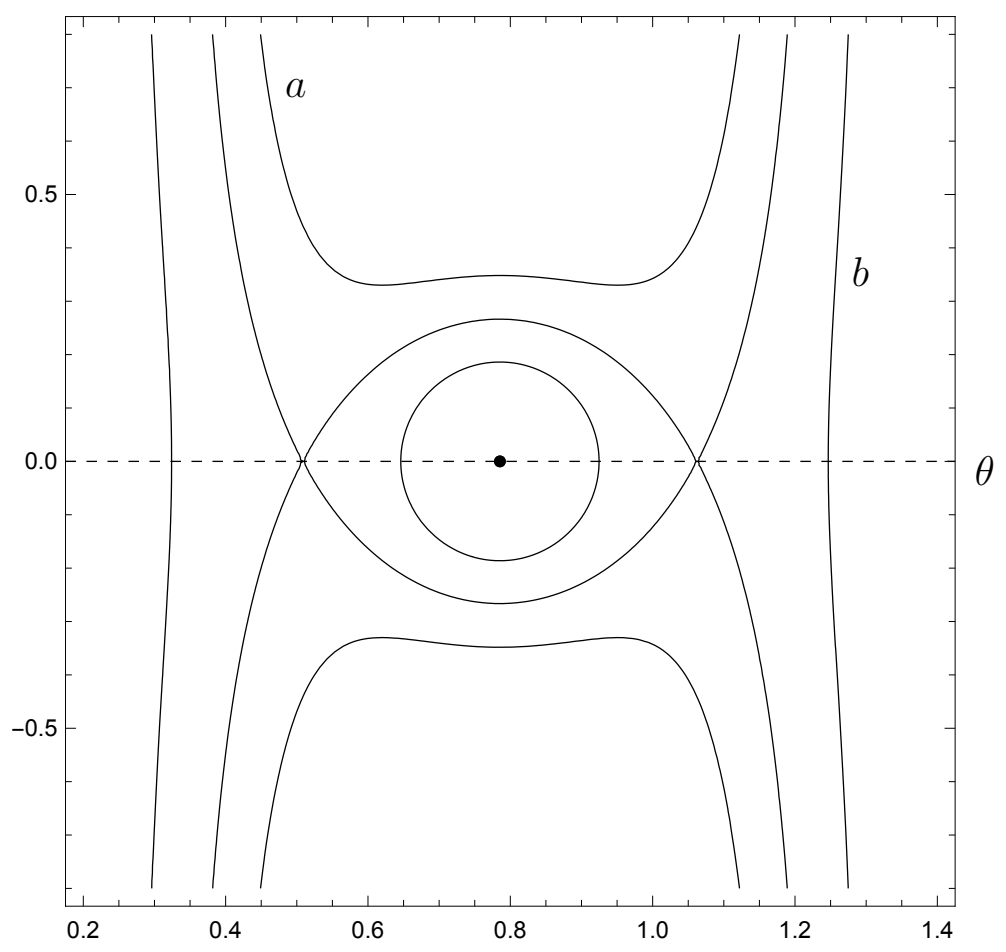

Figure 1: $\beta=1$ 

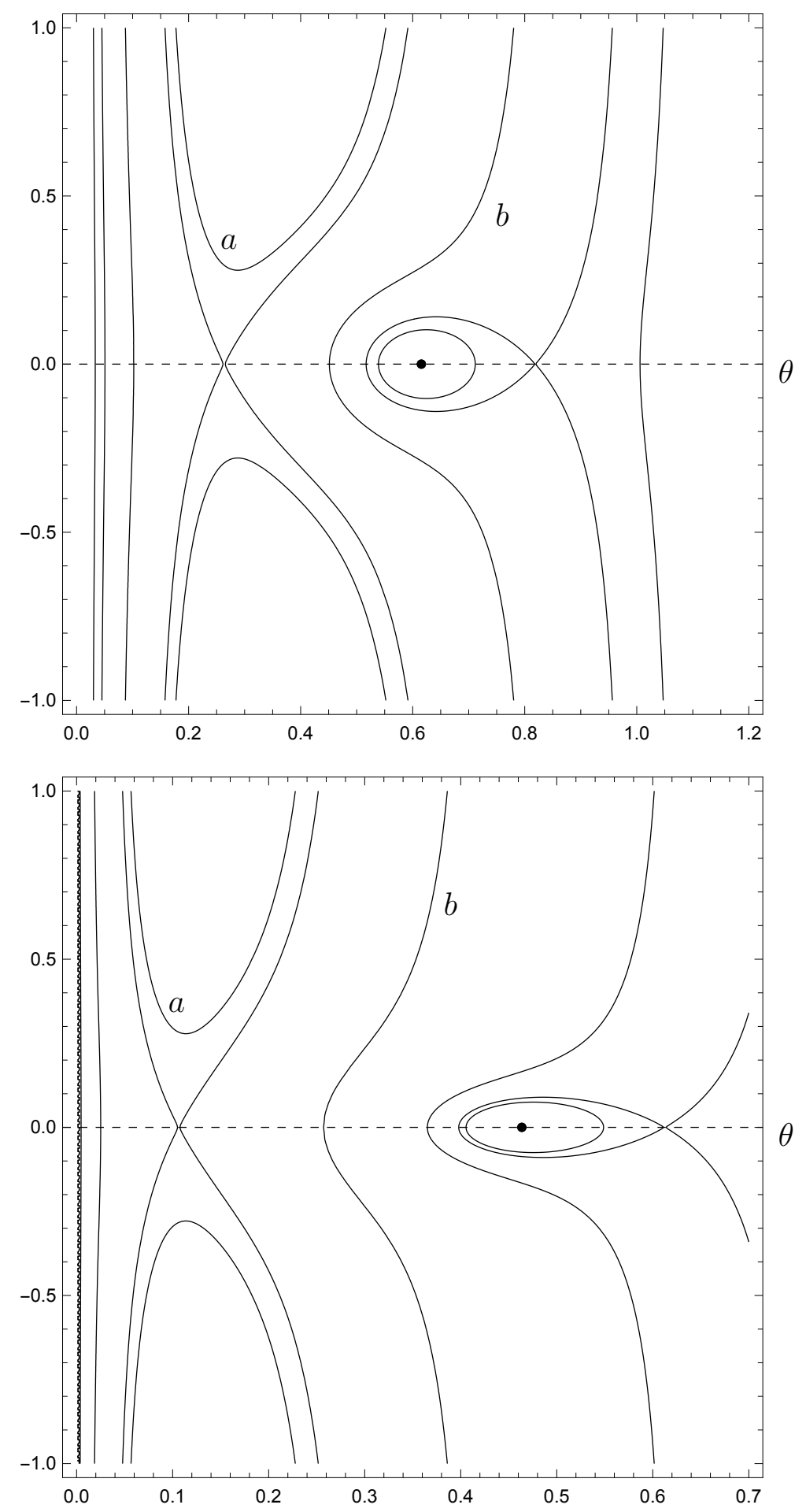

Figure 2: top: $\beta=2$, bottom: $\beta=4$ 
The dashed line is the $\theta$-axis, the vertical axis is the $W$-axis, and the black dot in each plot is the point $\left(\theta_{\beta}, 0\right)$. The phase portraits suggest that there are three possible types of motion: leapfrogging, single passage, and repulsion.

Leapfrogging motion are motions corresponding to closed orbits, which its existence was rigorously proved in Section 3. We can see that in each of the three portraits, there are closed orbits revolving around the point $\left(\theta_{\beta}, 0\right)$.

Single passage is the motion in which one filament goes through the other once and then separate from each other indefinitely. The orbits labeled $b$ correspond to such motion.

Repulsion is the motion in which one filament approaches the other until some minimal distance is attained, and then is repulsed away from each other. Orbits labeled as a correspond to such motion.

These dynamics qualitatively agree with the dynamics obtained in [2], supporting the validity of our model.

\subsection{Concluding Remarks}

We derived a PDE model describing the interaction of a pair of vortex filaments. The system was explicitly solved for a pair of straight and parallel lines, and showed that the motion resembled that of a pair of point vortices. We also proved rigorously that there exist solutions which correspond to leapfrogging motion of interacting coaxial circular filaments, and gave necessary and sufficient conditions for such motion to occur.

Although the model system (2.2) is a system of partial differential equations, the analysis carried out in this paper is essentially for systems of ordinary differential equations. As the next step, the author would like to consider the unique solvability of (2.2) in a mathematically rigorous setting, in a neighbourhood of exact solutions obtained in this paper at the very least, to investigate the stability of these solutions under non-symmetric perturbations.

\section{Acknowledgements}

This work was supported in part by JSPS Grant-in-Aid for Young Scientists(B) grant number $15 \mathrm{~K} 17579$.

\section{References}

[1] R.J. Arms and F.R. Hama, Localized-induction concept on a curved vortex and motion of an elliptic vortex ring, Phys. Fluids, 8 (1965), no.4, pp. 553-559.

[2] A.V. Borisov, A.A. Kilin, and I.S. Mamaev, The Dynamics of Vortex Rings: Leapfrogging, Choreographies and the Stability Problem, Regul. Chaotic Dyn., 18 (2013), no.1-2, pp. 33-62. 
[3] D.G. Crighton, Jet noise and the effects of jet forcing, In: The Role of Coherent Structures in Modelling Turbulence and Mixing. Lecture Notes in Physics, 136 (1981). Springer, Berlin, Heidelberg.

[4] L.S. Da Rios, Sul moto d'un liquido indefinito con un filetto vorticoso di forma qualunque [in Italian], Rend. Circ. Mat. Palermo, 22 (1906), no. 3, pp. 117-135.

[5] F. Dyson, The Potential of an Anchor Ring, Philos. Trans. Roy. Soc. London Ser. A, 184 (1893), pp. 43-95.

[6] F. Dyson, The Potential of an Anchor Ring - Part II, Philos. Trans. Roy. Soc. London Ser. A, 184 (1893), pp. 1107-1169.

[7] Y. Fukumoto, Higher-order asymptotic theory for the velocity field induced by an inviscid vortex ring, Fluid Dynam. Res., 30 (2002), no. 2, pp. 65-92.

[8] H. Helmholtz, Über Integrale der hydrodynamischen Gleichungen, welche den Wirbelbewegungen entsprechen, J. Reine Angew. Math., 55 (1858), pp. 25-55.

[9] W. M. Hicks, On the mutual threading of vortex rings, Proc. R. Soc. Long. A, 102 (1922), pp. 111-131.

[10] M. W. Hirsch and S. Smale, Differential Equations, Dynamical Systems, and Linear Algebra, 1974, Academic Press.

[11] A.K.M.F. Hussain and K.B.M.Q. Zaman, Vortex pairing in a circular jet under controlled excitation. Part 2. Coherent structure dynamics, J. Fluid Mech., 101 (1980), no. 3 , pp. $493-544$.

[12] R. Klein, A.J. Majda, and K. Damodaran, Simplifed equations for the interaction of nearly parallel vortex filaments, J. Fluid Mech., 288 (1995), pp. 201-248.

[13] Y. Murakami, H. Takahashi, Y. Ukita, and S. Fujiwara, On the vibration of a vortex filament [in Japanese], Applied Physics Colloquium [in Japanese], 6 (1937), pp. 1-5.

[14] P. K. Newton, The N-Vortex Problem, Applied Mathematical Sciences, 145 (2001), Springer-Verlag New York.

[15] P.G. Saffman, The Velocity of Viscous Vortex Rings, Studies in Appl. Math., 54 (1975), no. 4, pp. 371-380.

[16] K. Shariff, A. Leonard, Vortex Rings, Annu. Rev. Fluid Mech., 24 (1992), pp. 235279 .

[17] H. Yamada and T. Matsui, Preliminary study of mutual slip-through of a pair of vortices, Phys. Fluids, 21 (1978), pp. 292-294. 
[18] K.B.M.Q. Zaman, Far-field noise of a subsonic jet under controlled excitation, J. Fluid Mech., 152 (1985), pp. 83-111.

\author{
Masashi Aiki \\ Department of Mathematics \\ Faculty of Science and Technology, Tokyo University of Science \\ 2641 Yamazaki, Noda, Chiba 278-8510, Japan \\ E-mail: aiki_masashi@ma.noda.tus.ac.jp
}

